\title{
The significance of drop non-sphericity in sprays
}

\author{
Rainer N. Dahms ${ }^{1}$ and Joseph C. Oefelein \\ Combustion Research Facility, Sandia National Laboratories, Livermore, CA 94551, USA
}

\begin{abstract}
This paper presents a new framework to model drop dynamics in Lagrangian sprays. The framework builds on the Taylor Analogy Breakup (TAB) model. Real-fluid (gas-liquid) thermodynamics applicable to multicomponent systems are combined with Gradient Theory to facilitate detailed calculations of drop surface tension forces, oscillations, and breakup processes. This is combined with a more detailed treatment of deforming drop dynamics to construct more accurate representations of the local interfacial exchanges of mass, momentum, and energy. The framework is derived using an energy balance equation that explicitly enforces drop momentum conservation during the breakup process. This facilitates development of a refined set of drop equations that address current shortcomings in the prediction of drop properties over a wide range of relevant breakup conditions. The resulting drag forces, evaporation, and heating rates deviate significantly from the predictions given by contemporary drop models used in modern simulations. These deviations are quantified using Large Eddy Simulation (LES) with a Lagrangian-Eulerian modeling approach. The analysis demonstrates how the model improvements in the new framework provides a more detailed representation of physical complexities that are largely neglected in modern studies.
\end{abstract}

Keywords: Drop Deformation; Drop Breakup; Lagrangian-Eulerian Spray Dynamics.

\section{Introduction}

Liquid injection in modern combustion systems typically results in drop dynamics that are not in equilibrium with the local gas-phase flow characteristics. Drops are therefore subject to aerodynamic forces that cause deformation, oscillations, and a variety of breakup events. It has long been recognized that such processes can be classified by the Weber number, which characterizes the balance of forces imposed between the fluid flow and surface tension, and the Ohnesorge number, which characterizes the

\footnotetext{
${ }^{1}$ Corresponding Author; Email: Rndahms@sandia.gov
}

(C) 2015. This manuscript version is made available under the Elsevier user license http://www.elsevier.com/open-access/userlicense/1.0/ 
balance between viscous forces relative to the drop inertia and surface tension. Using these parameters, widely accepted regime diagrams for drop deformation and breakup have been compiled such as those derived by Hsiang and Faeth ${ }^{42}$ and Lasheras and Hopfinger. ${ }^{50}$ These diagrams classify the response of a single drop based on the local fluid dynamics and its thermodynamic state. At low Weber numbers, liquid drops deform and may undergo harmonic oscillations without breakup. At higher Weber numbers, critical drop distortions develop that result in bag or multi-mode breakup processes. At even higher Weber numbers, breakup becomes less governed by large scale drop dynamics and is instead determined by surface stripping or drop shattering processes. The classification of deformation and breakup processes is relatively uniform over a wide range of Ohnesorge numbers. At high Ohnesorge numbers, however, respective regime separators progressively shift to higher Weber numbers.

A variety of models have been developed to simulate deforming drop dynamics over the past several decades. Two models in particular have found wide acceptance and are used extensively in both academic and commercial flow solvers. ${ }^{2,6,44,57,91}$ The first is the WAVE model originally developed by Reitz, ${ }^{80}$ and Reitz and Diwakar. ${ }^{82}$ The second is the Taylor-Analogy-Breakup (TAB) model originally developed by O'Rourke and Amsden. ${ }^{75}$ The WAVE breakup model relates the mechanism of drop breakup with the analogy to Kelvin-Helmholtz instabilities along cylindrical viscous liquid jets. It assumes that the time of breakup and related drop sizes are proportional to the growth rate and wavelength of the most unstable Kelvin-Helmholtz instability. ${ }^{80,81}$ Past studies have also included the Rayleigh-Taylor mechanism to predict the surface growth rate, which determines drop breakup. ${ }^{46,83}$ Most recently, this model has been improved by extending the analogy to a jet with a linear shear layer in the surrounding gas-phase to eliminate the previous assumption of a velocity discontinuity at the fluid interface. ${ }^{110}$ The improved model includes the thickness of the shear layer in addition to the surface tension force as a leading order mechanism to drive surface wave instabilities. However, it neglects large scale drop deformations and as a consequence neglects the effects of these dynamics on drop mass, momentum, and energy transfer. In addition, the WAVE model assumes a constant drop size after breakup, which is not necessarily consistent with experimental evidence.

In contrast to the WAVE model, the TAB model is built on Taylor's analogy between drop dynamics and a forced spring-mass-damper system. ${ }^{107}$ It explicitly models the deformation and oscillation of drop dynamics and assumes that drop breakup occurs as soon as oscillations of the drop poles meet at its center. Then, an energy equation is used to predict the properties of the newly created drops. The model is conceptually valid for a wide range of Ohnesorge and Weber numbers as long as surface 
stripping phenomena do not significantly contribute to the breakup process. However, since its original introduction, many studies have shown that the predicted drop sizes after breakup are often under predicted. Also, deficiencies in the prediction of the spray angle exist, which the breakup model contributes to. ${ }^{48,55,104-106,109}$ Attempts to correct these deficiencies and extend the validity of TAB model to higher Weber number regimes have motivated further developments such as the Enhanced Taylor Analogy Breakup (ETAB) model. ${ }^{104,106}$

More recently, a stochastic modeling concept has been developed in which Kolmogorov's discrete model of breakup is solved as a differential Fokker-Planck equation for the pdf of droplet radii. $3,34,35$ To-date, most models used for device-scale spray simulations neglect the coupling of drop distortions, oscillations, interface dynamics, and breakup with the governing conservation equations. One exception is the dynamic drag model proposed by Liu et al. ${ }^{56}$ This model has been widely applied to account for the effect of drop distortion on its aerodynamic drag. ${ }^{56,91,96,108}$ However, it is only applicable to distortions for oblate spheroids. The deformation of prolate spheroids, which naturally occur during drop oscillations, is neglected. Further, the dynamic drag law is facilitated by assuming a linear relationship between the drag of a sphere and the drag of a sharp edged disc via a "distortion parameter." Recent studies have raised questions about the validity of this assumption over relevant ranges of drop Reynolds numbers. ${ }^{30,36,37,41,84}$ Viscosity effects are also neglected on drop deformation as well as the effects of internal flow dynamics on heat and mass transfer. ${ }^{10,13-19,29,65,90,91,114,116}$

Although Sirignano and co-workers were one of the first to quantify the effects of internal flow dynamics on drop heating and evaporation, ${ }^{1,92,93}$ such models are not fully utilized in current modeling frameworks. Furthermore, a perfect spherical drop represents one of the most fundamental assumptions in classic works on drop transport. ${ }^{25-27,51,78,94,100,101}$ This idealized reference configuration is also utilized in the derivation of classic breakup models. ${ }^{75,104,106}$. Further details on spray modeling and simulation are given by Gorokhovski and Herrmann ${ }^{33}$ and Ashgriz. ${ }^{4}$

The current work derives and investigates a Lagrangian model framework designed to facilitate detailed calculations of drop surface tension forces, oscillations, and breakup processes. This is combined with a more detailed treatment of deforming drop dynamics to construct more accurate representations of the local interfacial exchanges of mass, momentum, and energy. The framework builds on the Taylor Analogy Breakup (TAB) model, and is derived using an energy balance equation that explicitly enforces drop momentum conservation during the breakup process. This facilitates development of a refined set of drop equations that address current shortcomings in the prediction of drop properties 
over a wide range of relevant breakup conditions. Real-fluid thermodynamics and mean-field molecular gas-liquid interface methods are used to provide more accurate representations of local exchange processes between liquid drops and the gas-phase. The resulting drag forces, evaporation, and heating rates deviate significantly from the predictions given by contemporary drop models used in modern simulations. These deviations are quantified using Large Eddy Simulation (LES) with a LagrangianEulerian modeling approach. The analysis demonstrates how the model improvements in the new framework provides a more detailed representation of physical complexities that are largely neglected in modern studies.

\section{Model formulation}

Development of the model framework is performed in the context of the Lagrangian-Eulerian approach developed by Oefelein. ${ }^{43,67-73,87}$ The Large Eddy Simulation (LES) technique is used to represent the gas-phase. The dynamics of liquid drops are tracked using a system of Lagrangian particle equations. This is coupled to a generalized treatment of thermodynamic and transport processes for hydrocarbon mixtures ${ }^{67}$ and combined with mean-field molecular gas-liquid interfacial structures to compute surface tension forces. ${ }^{12}$ The foundational models that characterize drop trajectories and exchange of mass, momentum, energy, and species between phases are initially based on classical models. This baseline system of models is then extended to quantify the effects of drop non-sphericity on drop drag, evaporation, and breakup. A further refinement of the breakup model is then presented that strictly enforces the local conservation of mass, momentum, kinetic, and surface energy associated with individual drops. The proposed model improvements are demonstrated to provide superior momentum conservation properties compared to present breakup models.

\subsection{Baseline Lagrangian Equations of Motion and Transport}

The baseline Lagrangian equations of motion and transport are derived based on the assumptions that:

1. The spray is dispersed and that collisions between drops are negligible.

2. Drops can be characterized by equivalent spherical diameters.

3. Drops are small enough to become entrained within the turbulent eddies.

4. Drop motions due to shear flow are negligible. 
5. Drop acceleration due to static pressure gradients, virtual mass, and Bassett forces is negligible.

Under these circumstances, the equations that govern instantaneous particle motion can be written as

$$
\begin{aligned}
\frac{d \mathbf{x}_{p}}{d t} & =\mathbf{u}_{p} \\
\frac{d \mathbf{u}_{p}}{d t} & =\frac{3}{4} C_{D} R e_{p} \frac{\mu}{\rho_{p} d_{p}^{2}}\left(\mathbf{u}-\mathbf{u}_{p}\right)
\end{aligned}
$$

Similarly, the equations that govern instantaneous particle mass and heat transfer processes can be written as

$$
\frac{d m_{p}}{d t}=-\dot{m}_{p}
$$

100

$$
m_{p} C_{p_{p}} \frac{d T_{p}}{d t}=h_{p} \pi d_{p}^{2}\left(T-T_{p}\right)-\dot{m}_{p} \Delta h_{v}
$$

The subscript $p$ in these equations denotes values associated with individual drops. The quantity $d_{p}$ represents the equivalent spherical diameter of a fluid particle with mass $m_{p}$; i.e., $d_{p}=\sqrt[3]{6 m_{p} / \pi \rho_{p}}$. The terms $C_{D}$ and $R e_{p}$ represent the drag coefficient and particle Reynolds number, respectively. The particle Reynolds number is defined as

$$
R e_{p}=\frac{\rho d_{p}}{\mu}\left|\mathbf{u}-\mathbf{u}_{p}\right|
$$

and terms $\dot{m}_{p}, h_{p}$, and $\Delta h_{v}$ in Eq. (3) and (4) represent the vaporization rate, heat transfer coefficient, and enthalpy of vaporization, respectively.

Equations (1) through (4) are evaluated using a fourth-order Runge-Kutta scheme with semiempirical models employed to quantify the drag coefficient, vaporization rate, and heat transfer coefficient. The drag coefficient is often evaluated using correlations of the form proposed by Putnam. ${ }^{76}$ This correlation describes the influence of pressure and viscous forces acting on the surface of spherical particles and is given as a function of the drop Reynolds number as

$$
C_{D}^{o}=\frac{24}{R e_{p}}\left(1+\frac{1}{6} R e_{p}^{\frac{2}{3}}\right) \quad R e_{p} \leq 10^{3}
$$

Based on drag measurements of free-falling, evaporating, and burning drops of various liquids in hot air from Eisenklam et al., ${ }^{20,21}$ Yuen and Chen have established that Eq. (6) remains valid for evaporating 
drops if the Reynolds number is properly scaled by the average gas film dynamic viscosity. ${ }^{117}$ The underlying drop boundary film theory was developed by Abramzon and Sirignano. ${ }^{1}$ The calculation is facilitated by the " $1 / 3$ " rule, established by Hubbard et al. ${ }^{45}$ According to this rule, the average gas film temperature $T_{a v}$ and mixture composition $x_{i, a v}$ is calculated as follows

$$
T_{a v}=T_{p}+\frac{1}{3}\left(T_{\infty}-T_{p}\right)
$$

and

$$
x_{i, a v}=x_{i, p}+\frac{1}{3}\left(x_{i, \infty}-x_{i, p}\right)
$$

Then, the average gas film viscosity $\mu_{a v}$ is calculated accordingly using the real-fluid thermodynamics framework presented in Sec. 2.2. This facilitates calculation of the effective Reynolds number

$$
R e=\frac{\rho d_{p}\left|u-u_{p}\right|}{\mu_{a v}}=\frac{\rho d_{p} V_{\text {slip }}}{\mu_{\text {av }}}
$$

Models developed to approximate the vaporization rate and heat transfer coefficient in Eqs. (3) and (4) are summarized by Faeth, Law, and Sirignano. ${ }^{25-27,51,94}$ These works provide in-depth reviews of the classical drop models beginning with those derived by Ranz and Marshall ${ }^{78}$ and Spalding. ${ }^{100,101}$ Further works include the developments of non-equilibrium evaporation models. ${ }^{61,74}$ The most widely used correlations for low-pressure drop dynamics are

$$
\begin{aligned}
\dot{m}_{d_{R e_{p}=0}} & =\pi \rho_{s} \mathcal{D}_{s m} d_{p} S h_{d_{R e_{p}=0}} \ln \left(1+B_{M}\right) \\
N u_{d_{R e_{p}=0}} & =\frac{2 \ln \left(1+B_{M}\right)^{L e^{-1}}}{\left(1+B_{M}\right)^{L e^{-1}-1}}
\end{aligned}
$$

The term $\dot{m}_{d_{R e_{p}=0}}$ represents the vaporization rate in a quiescent environment. Terms $S h_{d_{R e_{p}=0}}$ and $N u_{d_{R e_{p}=0}}$ represent the corresponding Sherwood and Nusselt numbers at quiescent conditions, respectively. The quantity $L e=\lambda_{s} / \rho_{s} C_{p} \mathcal{D}_{s m}$ represents the Lewis number, where the subscript $s$ refers to the vapor mixture at the drop surface. The quantity

$$
B_{M}=\frac{Y_{p_{s}}-Y_{p_{\infty}}}{1-Y_{p_{s}}}
$$

represents the Spalding transfer number, where $Y_{p_{s}}$ and $Y_{p_{\infty}}$ represent the mass fraction of the drop 
phase at its surface and within the ambient fluid, respectively. The Spalding transfer number is evaluated using the relation

$$
\ln \left(1+B_{M}\right)=L e \ln \left(1+B_{T}\right)
$$

where for the low-pressure case

$$
B_{T}=\frac{C_{p}\left(T_{\infty}-T_{s}\right)}{\Delta h_{v}}
$$

Equations (12) through (14) provide the functional relationship between the surface temperature $T_{s}$ and mass fraction $Y_{p_{s}}$.

To account for convective effects, the multiplicative correction factors proposed by Faeth and Lazar $^{28}$ are employed. These factors take the form

$$
\begin{aligned}
\frac{\dot{m}_{p}}{\dot{m}_{d_{R e_{p}}=0}} & =1+\frac{0.278 R e_{p}^{1 / 2} S c^{1 / 3}}{\left[1+1.232 / \operatorname{Re}_{p} S c^{4 / 3}\right]^{1 / 2}} \\
\frac{h_{p}}{h_{d_{R e_{p}=0}}} & =1+\frac{0.278 \operatorname{Re}_{p}^{1 / 2} \operatorname{Pr}^{1 / 3}}{\left[1+1.232 / \operatorname{Re}_{p} \operatorname{Pr}^{4 / 3}\right]^{1 / 2}}
\end{aligned}
$$

where $S c=\mu_{s} / \rho_{s} \mathcal{D}_{s m}$ and $\operatorname{Pr}=\mu_{s} C_{p} / \lambda_{s}$ represent the Schmidt and Prandtl numbers, respectively.

\subsection{Thermodynamic, Transport, and Surface Tension Properties}

\section{The Lagrangian system of equations described above and subsequently modified below require detailed} treatment of thermodynamic and transport properties for the multicomponent systems of interest. In addition, accurate calculations of surface tension forces are required. In this study, the extended corresponding states model ${ }^{52,86}$ is employed using a modified Benedict-Webb-Rubin (BWR) equation of state to evaluate the PVT behavior of the inherent dense multi-component mixtures. Use of modified BWR equations of state in conjunction with the extended corresponding states principle has been shown to provide consistently accurate results over the widest range of pressures, temperatures and mixture states, especially at saturated conditions. Having established an analytical representation for real mixture PVT behavior, thermodynamic properties are obtained in two steps. First, respective component properties are combined at a fixed temperature using the extended corresponding states methodology to obtain the mixture state at a given reference pressure. A pressure correction is then applied using departure functions of the form given by Reid et al. ${ }^{79}$ Chapter 5 . These functions are exact relations derived using Maxwell's relations (see for example VanWylen and Sonntag ${ }^{112}$ Chapter 10) and make use of the real mixture PVT path dependencies dictated by the equation of state. 
Standard state properties are obtained using the databases developed by Gordon and McBride ${ }^{32}$ and

Kee et al. ${ }^{47}$ Chemical potentials and fugacity coefficients are obtained in a similar manner. Likewise, viscosity and thermal conductivity are obtained using the extended corresponding states methodologies developed by Ely and Hanley. ${ }^{22,23}$ Mass and thermal diffusion coefficients are obtained using the methodologies outlined by Bird et al. ${ }^{7}$ and Hirschfelder et al. ${ }^{40}$ in conjunction with the corresponding states methodology of Takahashi. ${ }^{103}$

Gradient Theory is applied to obtain accurate predictions of surface tension forces. Gradient Theory provides a widely accepted methodology to accurately calculate surface tension forces between multi-component gases and liquids. ${ }^{54,62}$ At equilibrium, the model has been shown to be equivalent to mean-field molecular theories of capillarity. The foundation of this theory was established by van der Waals in $1893^{85,111}$ and reformulated later by Cahn and Hilliard in $1958 .{ }^{9}$ Over the past decades, Gradient Theory has been successfully applied to a wide variety of fluids: hydrocarbons and their mixtures, polar compounds and their mixtures, polymer and polymer melts, vapor-liquid and liquid-liquid interfaces. Recently, Gradient Theory has also been successfully compared to Monte Carlo molecular simulations of vapor-liquid and liquid-liquid interfaces. ${ }^{60,63,66}$ There, Gradient Theory proved successful in capturing both surface tension and details of vapor-liquid molecular interfacial structures. In this work, Linear Gradient Theory is adopted which assumes a linear dependence of the interfacial distribution of each species with respect to the reference liquid-phase species. Linear Gradient Theory has proved to be successful in calculating binary and multi-component interface states of the kind considered here. ${ }^{89,119}$ The influence parameter, which determines the response of the density gradient to local variations of the species chemical potentials from their bulk value, is assumed to be density-independent ${ }^{59}$ and is calculated based on the prior works of Lin et al. ${ }^{54}$ For temperatures higher than the critical temperature of the vapor component, the corresponding reduced temperature is set to a single value, consistent with suggestions from Zuo and Stenby. ${ }^{119}$ This method has found wide acceptance and has also been adopted here ${ }^{89}$ even though more suitable and generalized methods have also been most recently proposed. ${ }^{12}$ The boundary conditions of the bulk vapor and liquid-phases for the interface calculation are obtained from vapor-liquid equilibrium calculations. 


\subsection{Modeling the Dynamics of Non-Spherical and Distorting Drops}

\subsubsection{The Taylor Analogy Breakup (TAB) Model}

To establish the proposed model improvements for treatment of drop distortion and breakup, this section provides a summary of the Taylor Analogy Breakup (TAB) model originally proposed by O'Rourke and Amsden. ${ }^{75}$ The TAB model assumes that simulations of drop deformations and oscillations can be facilitated by a forced, one-dimensional mass-spring-damper surrogate model. ${ }^{107}$ Drop oscillations are described by considering only the first fundamental deformation mode of an oscillating sphere. Higher modes are neglected. Over-damped conditions result in drop deformations, while under-damped drop dynamics result in harmonic oscillations with an amplitude and frequency that depend on the fluid dynamic forcing and systems natural resonant frequency and damping. Drop breakup is assumed to occur when oscillations of the drop poles meet at its center.

The one-dimensional forced mass-spring-damper system is described by ${ }^{75}$

$$
\frac{d^{2} y}{d t^{2}}=\frac{C_{F}}{C_{b}} \frac{\rho}{\rho_{p}} \frac{V_{s l i p}^{2}}{r_{p}^{2}}-\frac{C_{K} \sigma}{\rho_{p} r_{p}^{3}} y-\frac{C_{d} \mu_{p}}{\rho_{p} r^{2}} \frac{d y}{d t}
$$

where $\rho$ and $\rho_{p}$ are the ambient gas and liquid drop density, $V_{\text {slip }}$ is the slip velocity between the gaseous flow and the drop translational motion, $r_{p}$ is the drop radius, $\sigma$ is the surface tension, and $\mu_{p}$ the liquid drop viscosity. The non-dimensional distortion of the drop poles is defined as

$$
y=\frac{x}{C_{b} r_{p}}
$$

where $x$ is the distance between the dynamic position of the drop pole and its original position in its spherical shape. These quantities are determined dynamically during the simulation. The first term on the right-hand-side of Eq. (17) represents the fluid dynamic force. The second and third term on the right-hand-side represent the liquid drop analogy to a spring and damper, respectively. Values for the coefficients $C_{F}, C_{K}, C_{d}$, and $C_{b}$ are listed in Table 1, as given by Lamb. ${ }^{49}$ These coefficients are chosen to match the known oscillation dynamics of inviscid $\left(C_{K}=8\right)$ and damped viscous drops $\left(C_{d}=5\right)$ and to match the experimentally verified critical Weber number of $W e_{\text {crit }}=12$ for low viscosity Newtonian liquids. Breakup cannot occur below the critical Weber number, which is consistent with the drop deformation and breakup regime diagram of Hsiang and Faeth. ${ }^{42}$

Using the criterion above, breakup is assumed to occur if the non-dimensional drop distortion $y$ 
Table 1: Modeling constants of the TAB model, rigorously determined by Lamb.

\begin{tabular}{|c|c|c|c|}
\hline $\mathrm{C}_{F}$ & $\mathrm{C}_{K}$ & $\mathrm{C}_{d}$ & $\mathrm{C}_{b}$ \\
\hline $1 / 3$ & 8 & 5 & 0.5 \\
\hline
\end{tabular}

exceeds unity. After integration and discretization of Eq. (17), the final form of the model is ${ }^{75}$

$$
y(t)=\mathrm{We}_{c}+\exp \left(-\frac{\Delta t}{t_{D}}\right)\left[\left(y_{0}-\mathrm{We}_{c}\right) \cos (\omega \Delta t)+\frac{1}{\omega}\left(\frac{d y_{0}}{d t}+\frac{y_{0}-\mathrm{We}_{c}}{t_{D}}\right) \sin (\omega \Delta t)\right]
$$

where $y_{0}$ and $d y_{0} / d t$ is the drop distortion and distortion velocity at the beginning of the time step. The remaining terms in Eq. (19) are assumed to be constant over each time step and are defined as follows. The reduced Weber number is denoted as

$$
\mathrm{We}_{c}=\frac{C_{F}}{C_{K} C_{b}} \mathrm{We}^{\star}
$$
accepted assumption that the critical Weber number indicates the level below which breakup does not occur $^{8,42}$

$$
\mathrm{We}^{\star}=\frac{\mathrm{We}}{1+1.077 \cdot O h^{1.6}}
$$

The Ohnesorge number is defined as

$$
O h=\frac{\mu_{p}}{\sqrt{\rho_{p} d_{p} \sigma}}
$$

where $d_{p}$ is the drop diameter. The Weber number is defined based on the radius of the drop as

$$
\mathrm{We}=\frac{\rho V_{s l i p}^{2} r_{p}}{\sigma}
$$

215

The term $t_{D}$ in Eq. (19) denotes the oscillation damping time, which is based on Eq. (17) and is given by

$$
t_{D}=\frac{2 \rho_{p} r_{d}^{2}}{C_{d} \mu_{p}}
$$


The drop oscillation frequency is then defined as

$$
\omega=\sqrt{C_{K} \frac{\sigma}{\rho_{p} r_{p}^{3}}-\frac{1}{t_{D}^{2}}}
$$

The final form of the equation for the drop distortion velocity is then given by ${ }^{75}$

$$
\frac{d y}{d t}=\frac{\mathrm{We}_{c}-y}{t_{D}}+\omega \exp \left(\frac{-\Delta t}{t_{D}}\right)\left[\frac{1}{\omega}\left(\frac{d y_{0}}{d t}+\frac{y_{0}-\mathrm{We}_{c}}{t_{D}}\right) \cos (\omega \Delta t)-\left(y_{0}-\mathrm{We}_{c}\right) \sin (\omega \Delta t)\right]
$$

Using these definitions, the corresponding breakup times can be determined.

The breakup time is defined as the period between the initiation of aerodynamic forces on an initially undistorted and non-oscillating drop and the time when drop breakup occurs $(y>1)$. In the low Weber number breakup regime, when the Weber number is of the order of the critical Weber number, the breakup time $t_{B}$ can be determined from Eq. (19) by assuming

$$
\omega \cdot t_{B}=\pi \quad \rightarrow \mathrm{We} \sim o\left(\mathrm{We}_{c}\right)
$$

which, with the already defined oscillation frequency of an unviscid liquid and $C_{K}=8$ results in

$$
t_{B}\left(\mathrm{We} \sim \mathrm{We}_{c}\right)=\frac{\pi}{2} \sqrt{\frac{\rho_{p} r_{p}^{3}}{2 \sigma}}
$$

This breakup time agrees with the experimentally observed breakup time in the bag breakup regime $\left(\mathrm{We}_{c}<\mathrm{We}<100\right) .{ }^{42}$ For high Weber numbers, drop breakup occurs almost immediately after the initiation of aerodynamic forcing. This corresponds to the following assumption for the breakup time

$$
\omega \cdot t_{B} \ll 1 \quad \rightarrow \mathrm{We} \gg 1
$$

Inserting this assumption into Eq. (19) results in the following for the breakup time

$$
t_{B}(\mathrm{We} \gg 1)=\sqrt{3} \frac{r_{p}}{V_{\text {slip }}} \sqrt{\frac{\rho_{p}}{\rho}}
$$

which agrees with the experimentally observed breakup time in the stripping breakup regime $(100<$ We $<350) .{ }^{42}$ Thus, the TAB model is valid over this particular range of Weber numbers for a wide range of Ohnesorge numbers. At higher Weber numbers, however, breakup is increasingly determined 
by surface stripping and drop shattering processes and the TAB forced spring-mass-damper system is not necessarily a meaningful surrogate model.

\subsubsection{Modeling Drag and Evaporation of Distorted Drops}

To improve model predictions, a framework is developed that accurately couples the TAB equations for drop distortion and oscillations to the equations for drop momentum, evaporation, heat transfer, and internal circulation. For drag, the baseline expression given by Eq. (6) is corrected to account for the induced fluid motion on both sides of the interface. As presented by Sirignano, ${ }^{92}$ this correction is based on the analytical solution of hydrodynamic forces on viscous spheres for creeping flows. The solution is given by

$$
C_{D, v i s, s p h}^{\star}=\frac{8}{R e}\left(\frac{3 \lambda+2}{\lambda+1}\right) \quad \rightarrow R e \ll 1
$$

The parameter $\lambda$ is defined as the viscosity ratio

$$
\lambda=\frac{\mu_{d}}{\mu_{g}}
$$

where $\mu_{p}$ and $\mu$ are the viscosities of the drop and of the ambient gas, respectively.

While numerous expressions for the drag coefficient of solid spheres $(\lambda \rightarrow \infty)$ and bubbles $(\lambda=0)$ exist, recent studies by Feng and Michaelides provide a consistent correlation that applies to finite viscosity ratios. ${ }^{30}$ Their correlation is utilized here to relax the underlying assumption of Eq. (6) of a rigid sphere and include the effect of fluid-fluid interface dynamics and internal drop flows at finite viscosity ratios. The equation

$$
C_{D, v i s, s p h}=\frac{2-\lambda}{2} C_{D, b}+\frac{4 \lambda}{6+\lambda} C_{D, 2} \quad(0 \leq \lambda \leq 2 ; 5<R e<1000)
$$

applies at low viscosity ratios. For higher viscosity ratios, the following equation is valid

$$
C_{D, v i s, s p h}=\frac{4}{\lambda+2} C_{D, 2}+\frac{\lambda-2}{\lambda+2} C_{D, s, s p h} \quad(2 \leq \lambda<\infty ; 5<R e<1000)
$$

and applies over the same Reynolds number range. Note that the Reynolds number is calculated from the 1/3-rule defined in Eq. (9) to account for the effects of evaporation.

Using the correlations above, the momentum coupling model is modified by relaxing the underlying 
assumption of a spherical drop shape. This is accomplished using insights gained from direct numerical simulations of flows around non-spherical objects performed by Richter and Nikrityuk, ${ }^{84}$ who derived a regression model that predicts 99.8 percent of the variance of the drag coefficient. The equation has the form

$$
\frac{C_{D}}{C_{D, v i s, s p h}}=\frac{0.21+\frac{20}{R e}\left(\frac{\ell}{d_{p}}\right)^{0.58}+\frac{6.9}{\sqrt{R e}}\left(\frac{\ell}{d_{p}}\right)^{-1.4}}{0.21+\frac{20}{R e}+\frac{6.9}{\sqrt{R e}}}
$$

where the reference drag coefficient of the viscous sphere $C_{D, v i s, s p h}$ is modified according to the spanwise length $\ell$ of the deformed drop. This is normalized by the diameter $d_{p}$ of the volume-equivalent sphere.

The deformation represented by Eq. (35) accurately resembles the corresponding distortion modeled by TAB. As a consequence, the spanwise length $\ell$ can be calculated from the normalized distortion $y$ given by Eq. (19) as follows

$$
\ell=2\left(r_{p}-x\right)=2 r_{p}\left(1-C_{b} y\right)
$$

with $x$ defined in Eq. (18). This alters the characteristic time scale of the instantaneous particle momentum given by Eq. (2), which assumes spherical drops. Note that these modifications do not enter into the TAB model since its constants have already been derived to match the measurable drop deformation and oscillation dynamics.

Corresponding models for the mass and energy equations are derived following the approach taken for the momentum coupling. The Reynolds, Schmidt, and Prandtl numbers are calculated using average gas film quantities (as defined above) to properly account for the effects of drop evaporation. The drop heat and mass transfer coefficients are then modified to include the effect of fluid-fluid interface dynamics and internal drop flow patterns. The correction for the effective Nusselt number for viscous spheres is given as follows based on the work of Feng and Michaelides ${ }^{30,88}$

$$
N u_{v i s, s p h}=\frac{2-\lambda}{2} N u_{b}+\frac{4 \lambda}{6+\lambda} N u_{2} \quad(0 \leq \lambda \leq 2 ; P e \leq 1000)
$$


with $\lambda$ already defined in Eq. (32). At high viscosity ratios, the following equation applies

$$
N u_{v i s, s p h}=\frac{4}{\lambda+2} N u_{2}+\frac{\lambda-2}{\lambda+2} N u_{s, s p h} \quad(2 \leq \lambda<\infty ; P e \leq 1000)
$$

The Nusselt number for a solid sphere $(\lambda \rightarrow \infty)$ is given by the equation ${ }^{30}$

$$
N u_{s, s p h}=0.852 P e^{1 / 3}\left(1+0.233 R e^{0.287}\right)+1.24-0.182 R e^{0.355}
$$

The Nusselt number for a sphere with an intermediate viscosity ratio $(\lambda=2)$ is given by ${ }^{30}$

$$
N u_{2}=0.64 P e^{0.43}\left(1+0.233 R e^{0.287}\right)+1.41-0.15 R e^{0.287}
$$

Here, the Peclet number $P e$ is based on gaseous fluid quantities and thermal conductivity as

$$
P e_{N u}=\operatorname{Pr} \cdot R e
$$

The Sherwood number is obtained using Eqs. (37) and (38) using a Peclet based on the mass diffusion coefficient as

$$
P e_{S h}=S c \cdot R e
$$

Using the relations above, the underlying assumption of a spherical drop is now lifted.

Richter and Nikrityuk. ${ }^{84}$ have identified the sphericity $\phi$ and the cross-wise sphericity $\phi_{\perp}$ as the main geometric parameters that account for variations of Nusselt numbers when a sphere is distorted in its spanwise direction. This spanwise direction is directly calculated by TAB model. Similarly to Eq. (35), the correction equation is given by

$$
\frac{N u}{N u_{v i s, s p h}}=\frac{1.76+0.55 \phi \operatorname{Pr}^{1 / 3} \sqrt{R e} \phi_{\perp}^{0.075}+0.014 \operatorname{Pr}^{1 / 3} \operatorname{Re}^{2 / 3}\left(\frac{\phi}{\phi_{\perp}}\right)^{7.2}}{1.76+0.55 \operatorname{Pr}^{1 / 3} \sqrt{R e}+0.014 \operatorname{Pr}^{1 / 3} \operatorname{Re}^{2 / 3}}
$$

where the sphericity $\phi$ is defined as

$$
\phi=\frac{4 \pi r_{p}^{2}}{\operatorname{Srfc}_{p}}
$$


and the cross-wise sphericity $\phi_{\perp}$ is defined as

$$
\phi_{\perp}=\left(\frac{r_{p}}{H}\right)^{2}
$$

285

$$
\text { time scales of drop heating and evaporation. Unlike the drag coefficient, which implicitly includes the }
$$
surface area alteration of the deforming drop, the Nusselt and Sherwood numbers represent surface- 
averaged values only. As a consequence, the coupling model for mass and energy is obtained as a function of the surface area change relative to that of the mass-equivalent drop sphere. The final expression is given as a correction factor to the original drop diameter of the spherical drop $d_{p}$ as follows

$$
d^{2}=d_{p}^{2} \cdot \frac{\operatorname{Srfc}_{d}}{\pi d_{p}^{2}}
$$

Finally, the governing equation for viscous and distorted drop mass transfer processes is given by

$$
\left.\frac{d m_{p}}{d t}\right|_{v i s, n s p h}=\left.\frac{d m_{p}}{d t}\right|_{s, s p h} \cdot \frac{S h}{S h_{s, s p h}} \frac{\operatorname{Srfc}_{d}}{\pi d_{p}^{2}}
$$

where $\left.\frac{d m_{p}}{d t}\right|_{s, s p h}$ is defined in Eq. (3). The corresponding heat transfer equation is corrected in the same manner using the Nusselt number modification. In the limit of rigid spherical drops, this framework exactly recovers the original correlations of drag, mass, and heat transfer.

The coupled equations of mass, momentum, and energy also feed back into the TAB model. The TAB equations explicitly depend on the dynamic drop radius, which depends on the drop temperature and evaporation rate. With changing temperature, the liquid drop properties and surface tension also change. Similarly, aerodynamic forces are calculated through coupling to LES. A suitable temporal resolution of the drop dynamics is required in order to accurately capture these complex interactions between the unsteady aerodynamic forces and the drop response. This temporal resolution is determined by considering the characteristic time scales of drop oscillations, drag, evaporation, and heating relative to the fluid flow time.

\subsubsection{A Refined Model for Drop Breakup}

In the original work of O'Rourke and Amsden, a theory for the determination of the drop properties after a breakup event $(y>1)$ was derived based on an energy balance of surface and kinetic energy between drops before and after breakup. ${ }^{75}$ The energy balance before breakup, $E_{b}$, is given by

$$
E_{b}=4 \pi r_{p}^{2} \sigma+K \frac{\pi}{5} \rho_{p} r_{p}^{5}\left[\left(\frac{d y}{d t}\right)^{2}+\omega^{2}\right]
$$

where $K=10 / 3$ is the experimentally obtained ratio of the oscillation and distortion energy and the energy of the fundamental mode. The first term on the right-hand side represents the surface energy 
and the second term is the kinetic energy. The energy balance, $E_{a}$, of drops after breakup can be obtained similarly

$$
E_{a}=4 \pi r_{p}^{2} \sigma \frac{r_{p}}{r_{32}}+\frac{\pi}{6} r_{p}^{5} \rho_{p}\left(\frac{d y}{d t}\right)^{2}
$$

Here, the Sauter mean radius is given by

$$
r_{32}=\frac{r_{V}^{3}}{r_{p}^{2}}
$$

where $r_{V}$ and $\mathrm{r}_{p}$ are the volume-equivalent and surface-equivalent drop radii, respectively. Equating these formulas leads to the final expression for the Sauter mean radius proposed by O'Rourke and Amsden $^{75}$

$$
r_{32}=\frac{r_{p}}{1+\frac{8 K}{20}+\left(\frac{6 K-5}{120}\right) \frac{\rho_{p} r_{p}^{3}}{\sigma}\left(\frac{d y}{d t}\right)^{2}}
$$

The velocities of newly created drops receive a velocity, $v_{n}$, normal to the original drop velocity vector

$$
v_{n}=A C_{b} r_{p} \frac{d y}{d t}
$$

where $A$ is a proportionality constant postulated to be $A=1$ and the constant $C_{b}$ is listed in Table 1 . The exact direction of the normal drop velocity vector is assigned by random sampling.

Since the original work from O'Rourke and Amsden, many studies have shown that the drop sizes after breakup are often significantly under-predicted and that deficiencies in the prediction of the spray angle, to which the breakup model contributes, exist. ${ }^{48,55,104-106,109}$ This deficiency, along with an attempt to extend the validity of TAB model to higher Weber number regimes, has motivated further developments. Most notable are the Enhanced Taylor Analogy Breakup (ETAB) model, ${ }^{104,106}$ the Cascade Atomization Breakup (CAB) model, ${ }^{105}$ and the hybrid T-BLOB/T-TAB model. ${ }^{108}$ All of these frameworks depend on the use of proportionality constants to empirically match desired drop size distributions.

In contrast to the model approaches outlined above, the model for drop breakup presented here is derived directly from the fundamental set of conservation equations for mass, momentum, kinetic energy, and surface energy. Thus, it does not rely on the use of proportionality constants. The starting 

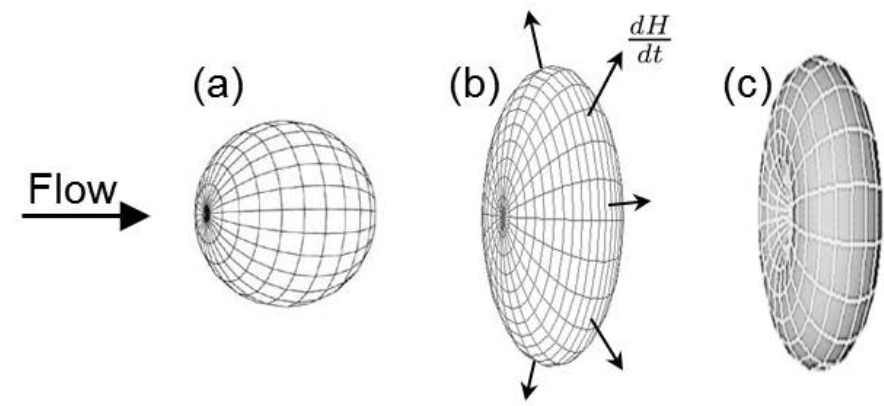

Figure 1: (a) The spherical shape of the drop is the underlying assumption of the original breakup energy equation, as given by the first term of the right-hand-side of Eq. (53). (b) The oblate shape of the drop at $y=1$. The velocity of the drop equator is denoted $\mathrm{dH} / \mathrm{dt}$. (c) The well-defined flattened torus shape of the drop at breakup $y=1$. In TAB, breakup is assumed to occur when the oscillation of the north and south pole meet at the center. ${ }^{75}$

point is a refined energy conservation equation similar to the one proposed by O'Rourke and Amsden, which was used to derive Eqs. (53) and (54). This equation is conditioned on the specific time of drop breakup and has no meaning at any other point in time. Figure 1 illustrates a sketch of different levels of drop distortions, which are fully consistent with the underlying TAB equations and assumptions. Figure 1 (a) shows a spherical drop without distortion or oscillations. This is the assumed drop state at breakup in the original energy equation, as given by the first term on the right-hand side of Eq. (53). Figure 1 (b) illustrates the distortion of the drop into an oblate shape, as predicted by TAB at the time of breakup $(y \geq 1)$. This shape can be obtained by a mass conservation equation as follows

$$
m=\text { const. }=\frac{4}{3} \pi \rho_{p} r_{p}^{3}=\frac{4}{3} \pi \rho_{p} L \cdot H^{2}
$$

where $L$ is half of the length of the minor axis (in flow direction) and $H$ is half of the length of the major axis of the oblate spheroid. The breakup model provides $L$ as a solution to Eq. (19) as

$$
L=r_{p}-x=r_{p}\left(1-C_{b} y\right)
$$

with $x$ defined in Eq. (18). Then, the major axis of the oblate spheroid can be calculated as

$$
H=\sqrt{\frac{r_{p}^{3}}{L}}
$$

According to the TAB's original criterion, the oscillation of the north and south pole of the drop meet at its center at the time of breakup. ${ }^{75}$ As a consequence, the shape of the drop further deforms into a 
flattened torus with a cross area of an ellipse as conceptually shown in Fig. 1 (c). The surface area of this shape is given by

$$
\operatorname{Srfc}_{b}=\pi \cdot H \cdot\left[\pi\left(\frac{H}{2}+b_{m}\right) \cdot\left(1+3 \frac{\lambda_{b}^{2}}{10+\sqrt{4-3 \lambda_{b}^{2}}}\right)\right]
$$

where $\lambda_{b}=\left(H / 2-b_{m}\right) /\left(H / 2+b_{m}\right)$ according to the approximate formula of the circumference of an ellipse proposed by Ramanujan. ${ }^{77}$ The term $H$ is the major axis of the underlying oblate spheroid at $y \geq 1$. This term is calculated using Eq. (60). The minor axis of the ellipse is obtained by the relation

$$
b_{m}=\frac{8}{3} \frac{r_{p}^{3}}{\pi H^{2}}
$$

This surface area can be related to a corresponding radius $r^{\star}$ of a sphere with the same area and to the physical radius $r$ of the mass-equivalent drop

$$
r^{\star}=r_{p} \sqrt{\frac{\mathrm{Srfc}_{b}}{4 \pi r_{p}^{2}}}=r_{p} \chi_{s}
$$

where $\chi_{s}$ is the square root of the ratio of the respective surface areas. This well-defined shape facilitates the calculation of the velocity magnitude of newly created drops as a function of the flow vector of the original drop. The velocity of the poles of the original drop, $d y / d t$, conditioned on the time of breakup, are given by Eq. (26). Mass conservation then allows the calculation of the respective normal velocity magnitude.

$$
\frac{d m}{d t}=0=\frac{d\left(\rho_{p} V\right)}{d t}=\frac{4}{3} \pi \rho_{p} \frac{d\left(H^{2} r_{p}\left(1-C_{b} y\right)\right)}{d t}
$$

where Eq. (59) is utilized and the time derivative of the liquid density is assumed to be negligible in comparison to the other terms ( $\rho_{p} \approx$ const). This leads to

$$
r_{p}\left(1-C_{b} y\right) \frac{d\left(H^{2}\right)}{d t}+H^{2} r_{p} \frac{d\left(1-C_{b} y\right)}{d t}=0
$$

It is important to note that $r_{p}$ is constant in this equation since it reflects the radius of the massequivalent sphere (see Eq. (59)). This results in the following expression

$$
2 H r_{p}\left(1-C_{b} y\right) \frac{d H}{d t}-C_{b} r_{p} H^{2} \frac{d y}{d t}=0
$$


which then is solved for the velocity magnitude normal to the flow velocity

$$
\frac{d H}{d t}=\frac{C_{b} H}{2\left(1-C_{b} y\right)} \frac{d y}{d t}=\frac{r_{p}}{2} \frac{C_{b}}{\sqrt{\left(1-C_{b} y\right)}} \frac{d y}{d t}
$$

From these equations, the proportionality constant in the original model (see Eq. (57)) is then calculated as follows

$$
A=\frac{1}{2\left(1-C_{b} y\right)^{1.5}}
$$

Note that for the special case of $C_{b}=0.5$ and $y=1$, that $A=\sqrt{2}$ which is similar to the originally postulated value of unity.

The energy balance for the drop mass before breakup, conditioned on the time of breakup, is given by the expression

$$
E_{b}^{\star}=4 \pi r_{p}^{2} \chi_{s}^{2} \sigma+K \frac{\pi}{5} \rho_{p} r_{p}^{5}\left[\left(\frac{d y}{d t}\right)^{2}+\left(\frac{C_{K} \sigma}{\rho_{p} r_{p}^{3}}-\frac{C_{D}^{2} \mu_{p}^{2}}{4 \rho_{p}^{2} r_{p}^{4}}\right) y^{2}\right]
$$

where the term $\chi_{s}$ is defined in Eq. (63). Thus, this equation includes damping of the drop in the calculation of the oscillation frequency, which is neglected in the original formulation. Note also that the distortion parameter is not set to $y=1$ since this value is directly linked to the critical Weber number (below which drop breakup does not occur). Under certain conditions such as elevated system pressures or non-Newtonian liquids, however, deviations from the classical value $\mathrm{We}_{c}=12$ may occur.

To obtain the energy balance for the drop mass after breakup, conditioned on the time of breakup, the kinetic energy of the drop mass in the normal flow direction must be calculated. This is given by

$$
E_{k i n}=\frac{1}{2} m_{p} v_{p}^{2}=\frac{1}{2} \frac{4}{3} \pi \rho_{p} r_{p}^{3}\left(\frac{d H}{d t}\right)^{2}
$$

Note that the kinetic energy is independent of the drop shape. Consulting Eqs. (67) and (68) leads to

$$
E_{k i n}=\frac{2}{3} \pi \rho_{p} r_{p}^{5} C_{b}^{2} A^{2}\left(\frac{d y}{d t}\right)^{2}
$$

Then, the energy balance of drops after breakup is defined as

$$
E_{a}^{\star}=4 \pi r_{p}^{2} \chi_{s}^{2} \frac{r_{p}}{r_{32}} \sigma+\frac{2}{3} \pi \rho_{p} r_{p}^{5} C_{b}^{2} A^{2}\left(\frac{d y}{d t}\right)^{2}
$$


Equating the energy distribution before (Eq. (69)) and after breakup (Eq. (72)) and solving for the Sauter mean radius $r_{32}$ then leads to

$$
r_{32}=\frac{r_{p}}{1+\frac{K}{20 \chi_{s}^{2}}\left(C_{K}-\frac{C_{D}^{2} \mu_{p}^{2}}{4 \sigma \rho_{p} r_{p}}\right) y^{2}+\left(\frac{K}{20}-\frac{A^{2} C_{b}^{2}}{6}\right) \frac{\rho_{p} r_{p}^{3}}{\chi_{s}^{2} \sigma}\left(\frac{d y}{d t}\right)^{2}}
$$

where $\chi_{s}$ is defined in Eq. (63), $\sigma$ is the surface tension, and $A$ is defined in Eq. (68). This equation is fully consistent with the underlying assumptions of the TAB model, but now includes a more realistic approximation of the surface energy, the kinetic energy of the newly created drops, and the true oscillation energy of drops calculated using Eq. (19) at the time of breakup. Aside from these modifications, the TAB modeling constants remain valid in all the equations above.

At the time of breakup, new drops are created with an initial location randomly chosen within the volume of the original distorted drop. The velocity $\vec{v}_{d}$ of each drop is initialized as follows

$$
\vec{v}_{p}^{\text {Init }}=\vec{v}_{o}+\operatorname{rand}\left(\vec{e}_{\perp}\right) \frac{d H}{d t}
$$

where $\vec{v}_{o}$ is the velocity vector of the original drop, $\operatorname{rand}\left(\vec{e}_{\perp}\right)$ is a random unit vector normal to $\vec{v}_{o}$, and $d H / d t$ is defined in Eq. (67). Note that Eq. (74), also applied in previous frameworks in a similar form, generally violates the conservation of momentum. The second term on the right-hand-side of Eq. (74) originates from the kinetic energy stored in the drop oscillation of the original drop before breakup. This oscillation has not contributed to the original drop momentum which solely resulted from its translational motion. After breakup, however, this oscillation is transformed into a translational motion of the new drops, which adds momentum as a consequence. In order to conserve momentum after breakup, the following equation is must be enforced

$$
m_{o} \vec{v}_{o}=\sum_{i=1}^{N} m_{i} \vec{v}_{i}^{\text {Init }}=\sum_{i=1}^{N} m_{i}\left[\vec{v}_{o}+\operatorname{rand}\left(\vec{e}_{\perp}\right) \frac{d H}{d t}\right]=m_{o} \vec{v}_{o}+\sum_{i=1}^{N} m_{i} \cdot \operatorname{rand}\left(\vec{e}_{\perp}\right) \frac{d H}{d t}
$$

Here, $N$ is the number of newly created drops and $m_{o}=\sum m_{i}$, which is the mass of the original drop. This expression immediately leads to the following condition for momentum conservation

$$
\sum_{i=1}^{N} m_{i} \cdot \operatorname{rand}\left(\vec{e}_{\perp}\right) \frac{d H}{d t}=0
$$


which is enforced in the following manner. First, the mass of each newly created drop $m_{i}$ is specified by determining the drop radius from sampling a suitable volume distribution function. This distribution is obtained from a proper application of the maximum entropy formalism as follows ${ }^{5,11}$

$$
f_{3}=q^{(q-4) / q} \frac{8 r_{i}^{3}}{D_{q 0}^{4} \Gamma(4 / q)} \exp \left(\frac{-\left(2 r_{i}\right)^{q}}{q D_{q 0}^{q}}\right)
$$

where $q$ is the order of constraint and $\Gamma$ is the gamma function. The parameter $q\left(q=1.39^{5}\right)$ is chosen assuming that the drop size distribution is independent of the velocity distribution. ${ }^{5}$ Usually, the imposed constraints include conservation of quantities such as surface energy, mass, or the partition of surface energy. Cousin et al. ${ }^{11}$ suggested, however, to determine the constraint based on a representative diameter of the drop distribution. It is recognized that $D_{q 0}$ can be obtained from the Sauter mean diameter $D_{32}$, defined in Eq. (73), as follows

$$
D_{32}=D_{q 0} \sqrt[q]{q} \frac{\Gamma(4 / q)}{\Gamma(3 / q)}
$$

This sampling of drop sizes proceeds until the total mass of all new drops has exceeded the mass of the original drop. This also determines the number of newly created drops $N$. Mass conservation between the new drops and the original drop is enforced by a subsequent scaling of all drop radii $r_{i}$ as follows

$$
r_{i}=r_{i, s}\left(\frac{m_{o}}{\sum m_{i, s}}\right)^{1 / 3}
$$

where $r_{i, s}$ is the drop radius from sampling and $m_{i, s}$ is the corresponding drop mass. Then, the velocities of drops initially prescribed by Eq. (74) are rotated in the plane normal to $\vec{v}_{o}$ to enforce momentum conservation. The resulting velocity of each drop is

$$
\vec{v}_{p}^{\star}=\vec{v}_{p}^{\text {Init }}-\frac{1}{m_{o}} \sum m_{i} \vec{e}_{i, \perp}^{0}
$$

where $\vec{e}_{\perp}^{0}$ is the drop normal velocity vector before rotation. The last term scales the rotational operation with the significance of the violation of momentum conservation as an inherent consequence of the initial solution obtained from Eq. (74). Without the rotation prescribed by Eq. (80), momentum conservation is violated in a non-negligible manner. The number of newly created drops, while depending on the computed ratio $r_{p} / r_{32}$, is generally insufficient to guarantee an approximate solution 
of Eq. (76) using statistical sampling. Furthermore, respective drop masses and drop velocities are chosen randomly and independently of each other. As a consequence, a large number of samples would be required for statistical convergence, which is conceptually not realizable by the physical process of drop breakup within breakup regimes predicted by the TAB model. While enforcing momentum conservation, however, Eq. (80) leads to a violation of energy conservation since it alters the velocity magnitude of each new drop without being constrained by the solution of Eq. (67). This inconsistency with the underlying assumptions of Eq. (73) is resolved through a subsequent scaling of all drop normal velocity magnitudes. We note from Eq. (76) that momentum remains conserved under such a scaling operation. Hence, the final velocity for each drop is determined as follows

$$
\vec{v}_{p}=\vec{v}_{o}+\left(\vec{v}_{p}^{\star}-\vec{v}_{o}\right) \cdot \sqrt{\frac{m_{o}(d H / d t)^{2}}{\sum m_{i}\left\|\vec{e}_{i, \perp}^{\star}\right\|^{2}}}
$$

where $\vec{e}_{i, \perp}^{\star}$ is the normal velocity vector of respective drops after the rotational operation prescribed by Eq. (80) is applied.

\section{Results and Discussion}

To demonstrate the model improvements derived above, the effects of drop non-sphericity on particle dynamics are now analyzed. The significance of these effects is established through comparisons of the characteristic rates for drop mass, momentum, and energy exchange with the flow between realistic distorted drops and volume-equivalent idealizations of drops as a perfect solid sphere. The corresponding deviation factors for drop drag, evaporation, and heating are defined as

$$
\chi_{C_{D}}=\frac{C_{D}}{C_{D, s, s p h}} ; \quad \chi_{\dot{m}_{p}}=\frac{\dot{m}_{p}}{\dot{m}_{p, s, s p h}} ; \quad \chi_{h_{p}}=\frac{h_{p}}{h_{p, s, s p h}}
$$

The subscript " $s, s p h$ " in the relations above refer to the reference models used for idealized spherical particle dynamics. The variation of these deviation factors quantifies the range of conditions under which the assumption of spherical drops becomes questionable.

The starting point for the discussion focuses on an analysis of drop shapes during imaged spray atomization. Figure 2 shows images of a water spray at ambient conditions performed by Marmottant and Villermaux. ${ }^{58}$ The injected liquid core after ligament breakup and drop formation is shown on the left. Figure 2 (middle) presents a magnified sample image for drop detection in a location downstream 

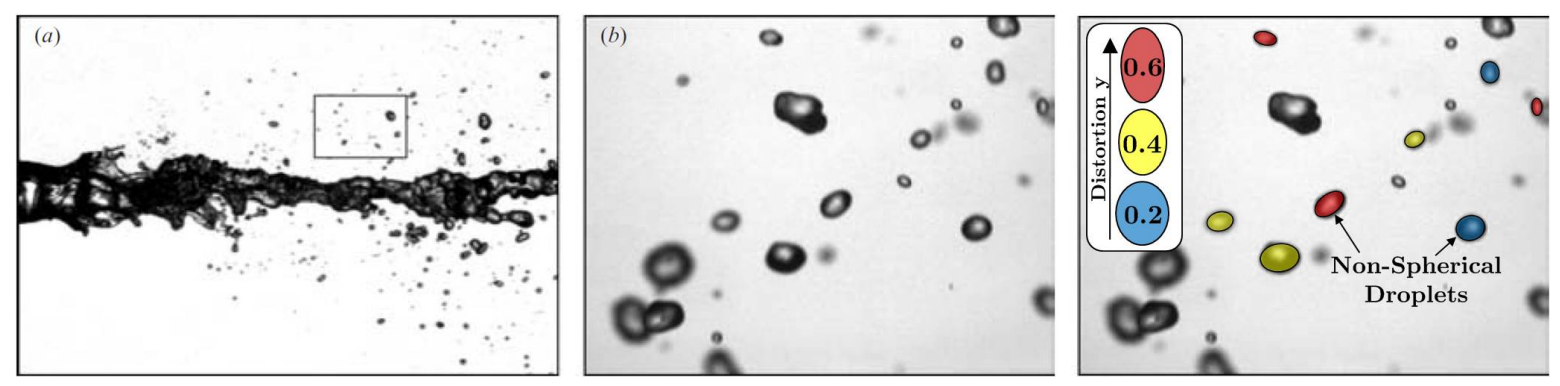

Figure 2: (Left) Image of a water spray after ligament breakup. (Middle) Sample image for drop detection. (Right) Same image as middle with added color coding to visualize selected drop distortions, as quantified using Eqs. (18), (59), and (60). Reprinted from "On spray formation," by P. Marmottant and E. Villermaux, Journal of Fluid Mechanics, Vol. 498, pp. 73-111, Copyright 2004 by Cambridge University Press. DOI: 10.1017/S0022112003006529

of the ligament breakup region. Marmottant and Villermaux reported to have selected this location in part to let the biggest drops relax towards a spherical shape. ${ }^{58}$ The actual state of the drop shapes, however, is obtained here using Eqs. (18), (59), and (60). Color coding is added in Fig. 2 (right) to visualize the drop distortion parameter for a selected set of representative drops. It is recognized from the analysis that many drops exhibit non-negligible shape distortions $\left(0.2<y<0.6 ; y_{\text {Sphere }}=0\right.$ and $y_{\max }=1$ ). It is also noteworthy that substantial shape distortions are present despite significant surface tension forces that exist between the water jet and the gaseous air $(\sigma \approx 70 \mathrm{mN} / \mathrm{m})$. Note that corresponding surface tension forces in liquid-fueled devices such as internal combustion engines (e.g., gas turbines or reciprocating) are generally lower, which may result in even more significant deformations than those shown here.

The model framework is exercised using LES to represent gas-phase quantities. Simulations are performed using the code framework RAPTOR, which is a fully compressible solver that employs non-dissipative, discretely conservative, staggered, finite-volume differencing. Temporal integration is performed using a dual-time multistage scheme, which facilitates time step settings based on physical accuracy considerations rather than numerical stability limitations. The subgrid-scale (SGS) closure is obtained using the "mixed" dynamic Smagorinsky model by combining the models proposed by Erlebacher, Hussaini, Speziale and Zang ${ }^{24}$ and Speziale ${ }^{102}$ with the dynamic modeling procedure. ${ }^{31,53,64,113,118}$ The Smagorinsky, SGS-Prandtl and SGS-Schmidt numbers are evaluated dynamically as functions of space and time. The overall model includes the Leonard and cross-term stresses and provides a Favre averaged generalization of the Smagorinsky eddy viscosity model ${ }^{95}$ combined with gradient diffusion models that simulate SGS mass and energy transport processes. Detailed 
documentation and representative case studies are given by Oefelein and co-workers. ${ }^{43,67-73,87}$

The calculations performed were selected to emulate realistic conditions that the system of models are expected to work under. A model gas turbine configuration based on previous research ${ }^{67,72}$ was used at conditions where experimental validation data was available (see Sommerfeld et al. ${ }^{97-99}$ ). This experiment provides detailed measurements of swirling particle-laden flow in a model combustion chamber that consists of a sudden pipe expansion with a centered (primary) and annular (secondary) jet discharging into a cylindrical test section. Experimental measurements were acquired using a onecomponent phase-Doppler-anemometer (PDA) to obtain mean and rms gas-phase and drop statistics of velocity and particle size. This experiment provides a good benchmark for validation of LES with well-defined boundary conditions, and also a good baseline to study the effects of drop deformation and breakup compared to the spherical particle dynamics measured in the experiment.

A schematic of the experimental apparatus and matching computational domain is shown in Fig. 3 (left). The rig is cylindrical and consists of an injector section, main chamber, and plenum chamber. The coaxial injector houses a centered particle-laden primary jet surrounded by a swirling annular secondary jet. Flow is injected into the main chamber from the top. Upon entering the chamber, the flow undergoes a sudden expansion followed by a series of complex fluid dynamic interactions. The flow then undergoes a second expansion at the exit of the main chamber into a plenum chamber. The region of interest showing the eight axial stations where cross-sectional PDA measurements were made is shown in Fig. 3 (right). Gas-phase and particle-phase mean and rms velocity components are given with simultaneous measurements of the particle size and mass flux distributions. Data were obtained with two different particle mass loadings in the primary jet, for three different particle size classes.

Particles are injected into the primary jet according to a distribution provided by measurements, as described in detail by Oefelein et al. ${ }^{72}$ This distribution produces a mean number diameter of $45 \mu \mathrm{m}$ and particle sizes over the range $20 \leq d_{p} \leq 80 \mu \mathrm{m}$. The walls inside the injector ducts are assumed to be adiabatic. Walls in the main chamber are assumed to be $300 \mathrm{~K}$. The geometry is characterized in dimensionless units using the radius $\mathrm{R}$ of the outer wall of the secondary jet as the reference length scale. Here, $R=32 \mathrm{~mm}$. The main chamber is approximately 30 dimensionless units long and extends a radial distance of approximately 3 dimensionless units. The injector section (not shown) extends 8 units upstream from the main chamber face plate. The plenum chamber begins approximately 30 units downstream of the main chamber, ends at 50 units, and extends a radial distance of 9 units. 

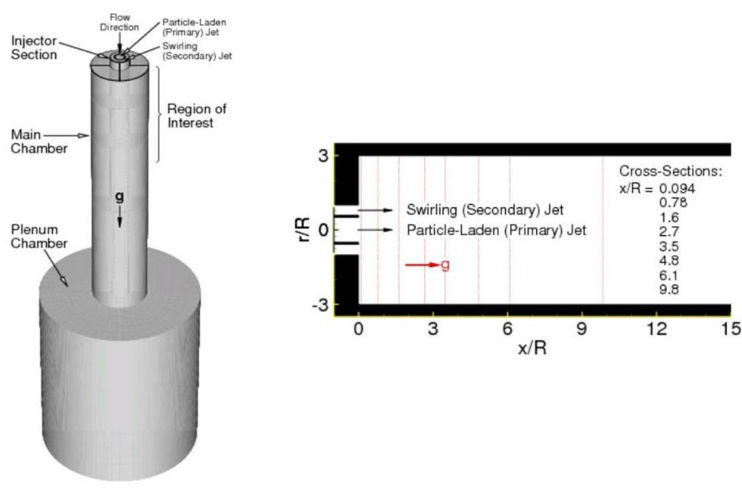

Figure 3: (Left) Schematic of the computational domain used for LES of the Sommerfeld et al. ${ }^{97-99}$ experiment. (Right) Region of interest with locations where cross-sectional PDA measurements were made.

The primary jet has a radius of $r / R=0.5$. Flow from the primary jet evolves to a fully-developed turbulent state prior to injection and enters the main chamber axially. The secondary jet extends over a radial interval of $0.59 \leq r / R \leq 1$, is fully-developed, and is injected into the main chamber with a swirling azimuthal velocity component.

Building on the results from the past validation studies, the model framework derived in Section 2 is used to analyze the injection of liquid $\mathrm{n}$-decane drops at a temperature of $\mathrm{T}_{p}=363 \mathrm{~K}$ into compressed air at $\mathrm{p}=10$ bar and $\mathrm{T}_{\text {air }}=475 \mathrm{~K}$. The goal is to use the LES to emulate realistic operating conditions under which evaporation and breakup processes occur. As a first step, the drop distribution inside the region of interest is mapped according to the drop distortion and breakup regime map of Hsiang and Faeth. ${ }^{42}$ Results are shown in Fig. 4 (left). This scatter plot quantifies the drop states at an arbitrary instant in time. While individual states generally vary over time, the characteristics of the envelope of all drop conditions is largely time-independent. The instantaneous drop positions and states showing the balance between the rate of injected liquid and evaporation is shown in Fig. 4 (right). Figure 4 (left) demonstrates that drops experience virtually all Weber numbers below We $\approx 70$. Hence, drop dynamics are governed by deformations $(\mathrm{We}<2)$, harmonic oscillations $(\mathrm{We}<12)$, multimode, and bag breakup processes $(\mathrm{We}<100)$. The corresponding range of Ohnesorge numbers is $0.01<\mathrm{Oh}<0.05$. Besides affecting drop oscillations (see highlighted region), Ohnesorge numbers do not significantly influence the identified drop dynamics. Hence, in what follow, the analysis focuses on corresponding effects of Weber numbers and Ohnesorge number effects are neglected.

The upper range of Weber numbers for which the TAB model remains applicable is also highlighted in Fig. 4 (left). Thus, the TAB model is valid under all conditions of the resulting envelope of drop 

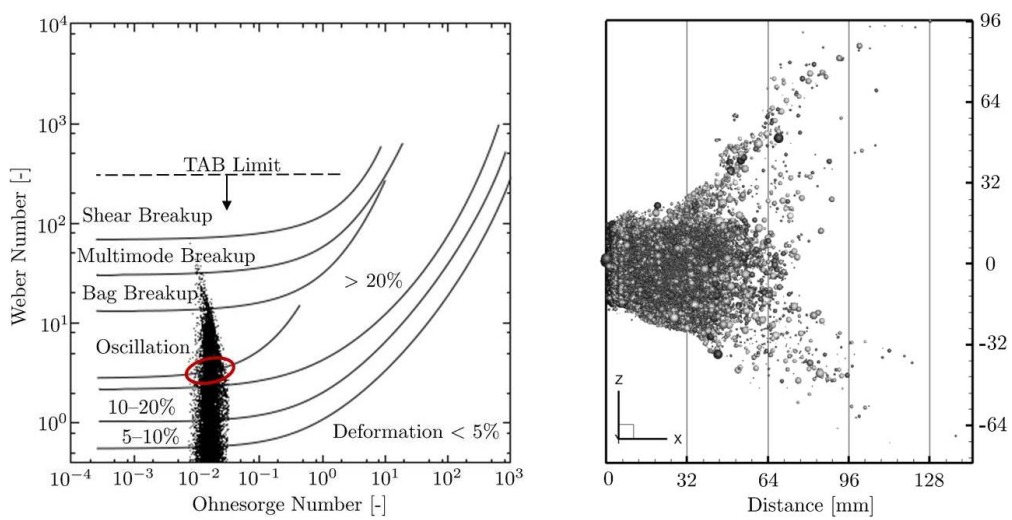

Figure 4: (Left) Drop distribution inside the region of interest mapped according to the drop distortion and breakup regime map of Hsiang and Faeth. ${ }^{42}$ Besides affecting drop oscillations (highlighted region), Ohnesorge numbers do not significantly influence the dynamics. The upper range of Weber numbers under which TAB model is applicable is also highlighted. (Right) Instantaneous drop positions and states showing balance between rate of injected liquid and evaporation.

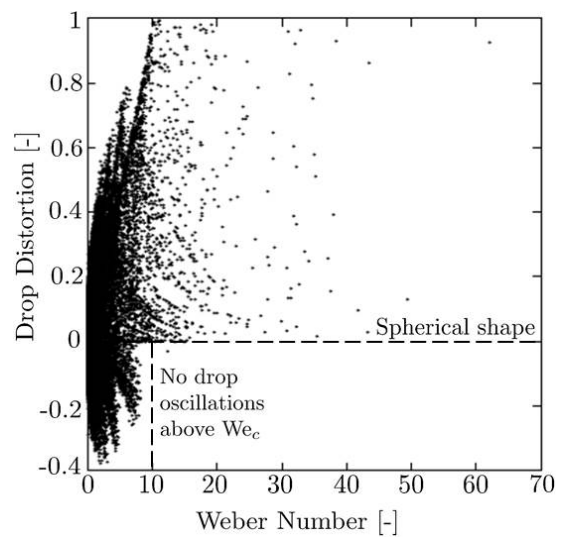

Figure 5: Drop distortions as a function of their local Weber numbers. Consistent with Fig. 4 (left), no drop oscillations are predicted by TAB model above the critical Weber number $\mathrm{We}_{c}$.

states. Thus, simulations of drop dynamics are performed using Eqs. (19) and (26), respectively. Figure 5 shows drop distortions, as defined in Eq. (18), as a function of the local drop Weber number. A drop distortion of $y=0$ represents a perfectly spherical drop. Positive and negative drop distortions

characterize drops that have been deformed into an oblate or prolate spheroids, respectively. Hence, negative drop distortions only occur during harmonic drop oscillations. Results demonstrate that no drop oscillations are predicted by the TAB model above the critical Weber number $\mathrm{We}_{c}$. At higher than critical Weber numbers, Fig. 5 illustrates that many drops exhibit significant shape deformations.

Figure 6 (left) shows drop drag coefficient corrections relative to the solid sphere reference due to the finite viscosity of the drop. This correction is calculated using Eq. (34), which depends on both the drop Reynolds number and viscosity ratio $\lambda$ between the liquid and gaseous phase (see Eq. (32)). 

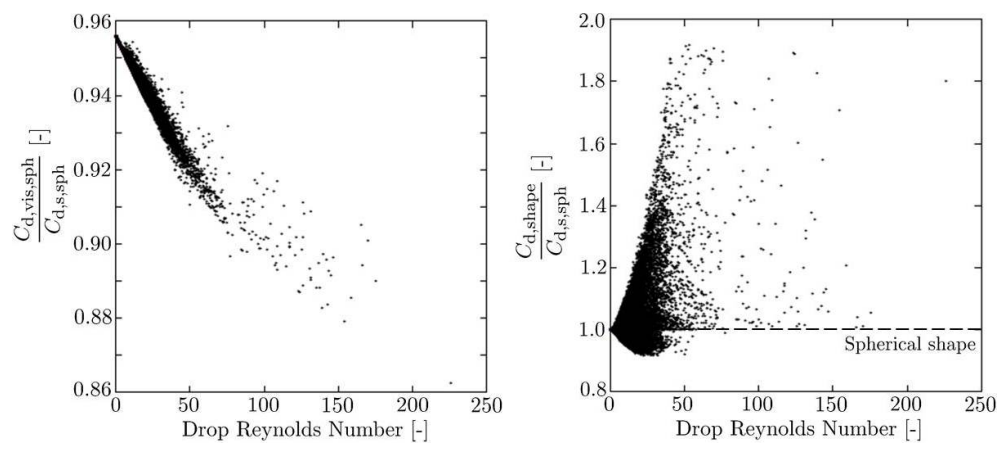

Figure 6: (Left) Finite drop viscosity drag correction to the solid sphere reference calculated using Eq. (34). (Right) Drop drag coefficient correction to the solid sphere reference due to shape distortion calculated using Eq. (35).

Under the conditions here, the viscosity ratio varies between values of $7<\lambda<16$. However, as shown in Fig. 6 (left), at small drop Reynolds numbers, the effect of varying viscosity ratios on the drag correction diminishes. Note also that a noticeable difference in drag coefficient correction persists $(\sim 0.96)$ even in the limit of small drop Reynolds numbers. This is consistent with observations that solid spheres $(\lambda \rightarrow \infty)$ generally exhibit higher drag coefficients than bubbles $(\lambda=0)$ regardless of the Reynolds number. Hence, the correction due to finite drop viscosity consistently lowers the drag coefficient in relation to the solid sphere reference.

Effects of drop distortions on corresponding drag corrections are shown in Fig. 6 (right). The transition to unsteady drag coefficients for spheres and ellipsoids are known to occur at Reynolds numbers of $R e>260 .{ }^{84}$ Here it is shown that the drag coefficient correction generally increases with an increase of drop Reynolds number. A strong positive correlation exists between the Reynolds number and the Weber number. Hence, higher Reynolds numbers also correlate with higher Weber numbers and, as a consequence, a larger degree of drop deformation is observed in Fig. 5. Drag coefficient corrections below unity indicate that drops have deformed into prolate spheroids. This physical complexity only occurs during drop oscillations, which are generally present below the critical Weber number, and thus result in only moderate deformations (see Fig. 5). Drag coefficient corrections generally increase with increasing Reynolds numbers. Since it was established that the range of physical realizable Reynolds numbers during drop oscillations is limited, their effect on the corresponding drag coefficient is also limited as a consequence. This explains, in combination with the moderate distortion amplitude in this regime $(y>-0.4)$, the trends observed in Fig. 6 (right), which show much more pronounced corrections for positive drop distortions at high drop Reynolds numbers.

Comparison of drag corrections due to finite drop viscosity and deformation using the new model 


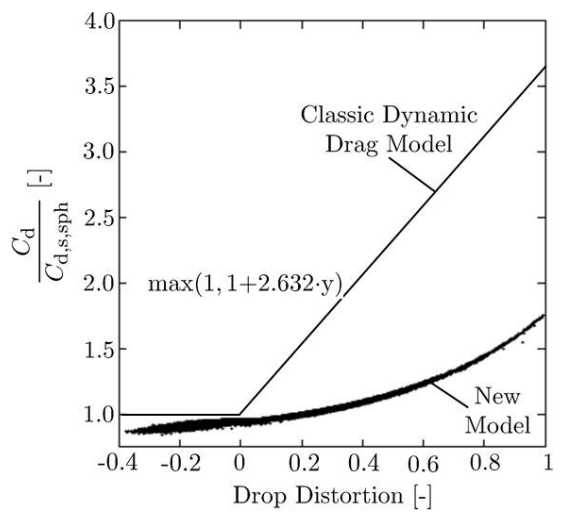

Figure 7: Comparison of drag corrections using the new model presented in Eq. (35) and the classic dynamic drag model proposed by Liu et al. ${ }^{56}$.

presented in Eq. (35) and the classic dynamic drag model proposed by Liu et al. ${ }^{56}$ is shown in Fig. 7. The observed scatter is related to variations in drop Reynolds number, Weber number, and viscosity ratio at constant drop distortion. The classic dynamic drag model proposed by Liu et al., ${ }^{56}$ which is often applied in industry and academia, ${ }^{56,91,96,108}$ is also shown for comparison. This model is given by the expression ${ }^{56}$

$$
\frac{C_{D}}{C_{D, s, s p h}}=\max (1,1+2.632 y)
$$

Note that this does not apply at negative drop distortions and it therefore entirely neglects the presence of prolate spheroid deformations. It also neglects the effects of fluid-fluid interface dynamics and internal flow field patterns which form in viscous drops. These effects tend to decrease the drop drag in comparison to the solid reference. The dynamic drag model also postulates the validity of a linear relationship of the drag coefficients with respect to the distortion parameter. This assumption is not necessarily supported by the results presented in Fig. 7. Finally, the dynamic drag model assumes that the drag coefficient at the critical drop distortion of $y=1$ can be approximated by the (measured) drag coefficient of a sharp-edged disc. However, this assumption significantly over-predicts the corresponding drag coefficient in comparison to the results of Eq. (35), which may be more fundamentally consistent with the underlying assumptions of drop deformations built into the TAB model.

Based on the detailed analysis presented in Fig. 7 and Eq. (35), the following simplified drop drag deformation model is proposed

$$
\chi_{C_{d}}=\frac{C_{d}}{C_{d, s, s p h}}=0.1 \cdot e^{2.25 y}+0.83
$$



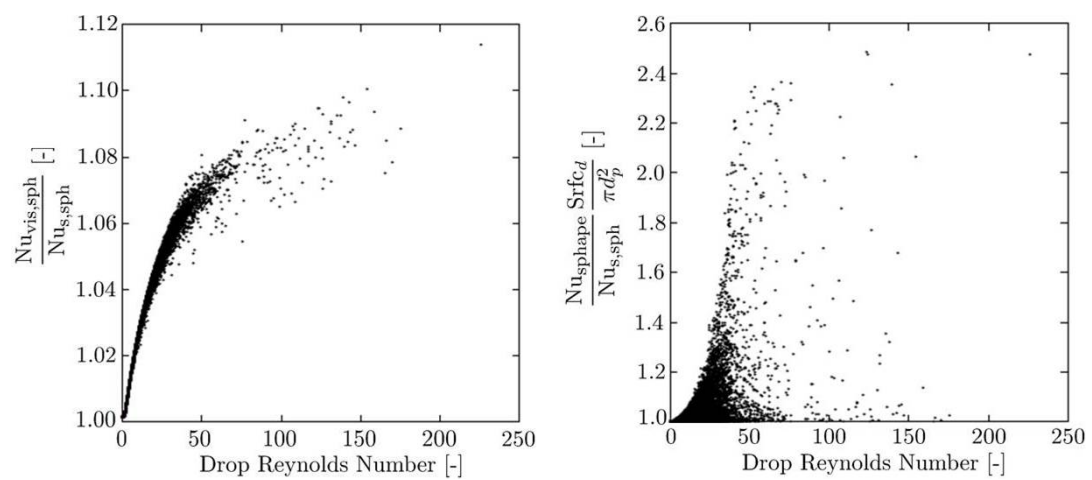

Figure 8: (Left) Drop viscosity corrections for the Nusselt number calculated using Eqs. (38) and (41). (Right) Drop distortion corrections of heating rates.

The model exhibits excellent agreement with the results from the detailed simulations, which included a meaningful range of viscosity ratios $(7<\lambda<16)$. It applies across the entire range of possible drop deformations $(-1 \leq y \leq 1)$. However, it must be noted that effects of extreme (and unlikely) prolate spheroid deformations $(y<-0.5)$ have not been established in this study and corresponding results from the model can only be viewed as a mathematical extrapolation.

Following the approach outlined above, Fig. 8 (left) shows finite drop viscosity corrections to Nusselt numbers in relation to the solid sphere reference as a function of drop Reynolds number. Corresponding corrections to the Sherwood number are very similar and therefore omitted here. These corrections have been calculated using Eq. (38), where the Peclet number is defined using the Prandtl number of n-decane (see Eq. (41)). Similar to the drag coefficient correction shown in Fig. 6 (left), differences in Nusselt numbers diminish at low drop Reynolds numbers. In contrast to the physical complexity observed in Fig. 6 (left), both Sherwood and Nusselt numbers approach the solid sphere reference values at low drop Reynolds numbers regardless of the viscosity ratio.

Figure 8 (right) shows drop distortion corrections (without consideration of finite drop viscosity effects) of drop heating rates to the solid sphere reference as a function of the drop Reynolds number. The analysis shows similar trends as already observed for the drag coefficient corrections shown in Fig. 6 (right). It is recognized that corresponding energy transfer rates are also enhanced at negative drop distortions for prolate spheroids. Similar to effects of finite drop viscosity, a strong correlation between both transfer rates exist. This is partly explained due to the significant effect of the surface area increase over the spherical reference shape. This increase affects both transfer rates in equal measure. The remaining deviations result from differences in Sherwood and Nusselt numbers due to shape distortions, as calculated by Eq. (43). Due to the larger Sherwood number correction, the 


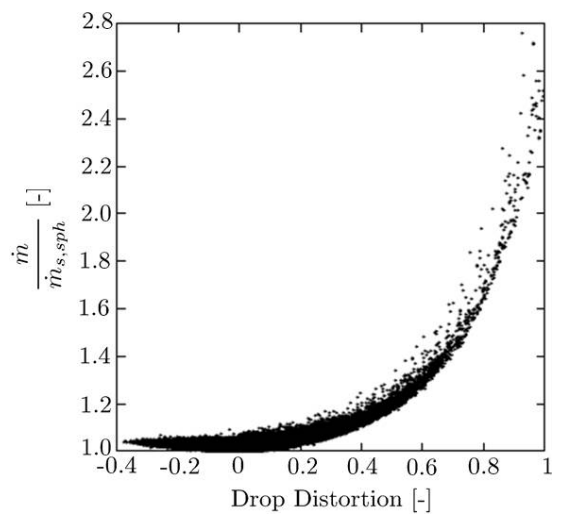

Figure 9: Effective evaporation rate corrections in relation to the solid sphere reference as a function of drop distortion, calculated based on Eq. (52).

corresponding correction to the evaporation rate is slightly higher than the respective correction to drop heating.

In analogy to Fig. 7, the effective correction to the evaporation rate relative to the solid sphere reference solution is calculated and shown in Fig. 9. Note that the respective correction to drop heating rates closely resembles this solution and is thus omitted here. Differences result from effects due to fluid-fluid interface dynamics, internal flow field patterns, and drop deformations. The observed scatter in the plot is related to variations in drop Reynolds number, Weber number, and viscosity ratio at constant drop distortion. These results demonstrate that consideration of drop coupling dynamics affect mass and energy transfer rates even more significantly than the drag forces. To account for this, the following simplified deformation model for drop evaporation and heating is proposed based on the analysis of Fig. 9 and Eq. (52)

$$
\chi_{\dot{m}_{p}} \approx \chi_{h_{p}}=0.12 y^{2}+0.02 \cdot e^{4.3 y}+1.02
$$

The model assumes an analogy between heat and mass transfer and is supported by the analysis presented in Fig. 8. It reproduces the average corrections for drop viscosity $(7<\lambda<16)$ and distortions shown in Fig. 9. The model formally applies across the entire range of possible drop deformations $(-1 \leq y \leq 1)$. However, it should again be noted that effects of extreme (and unlikely) prolate spheroid deformations in flow direction $(y<-0.5)$ have not been established in this study and corresponding results from this model can also only be viewed as a mathematical extrapolation.

Figure 10 now shows a visualization from LES of the instantaneous spray distribution with drops color-coded by their drop Reynolds number (top); the effective drop drag coefficient correction calcu- 


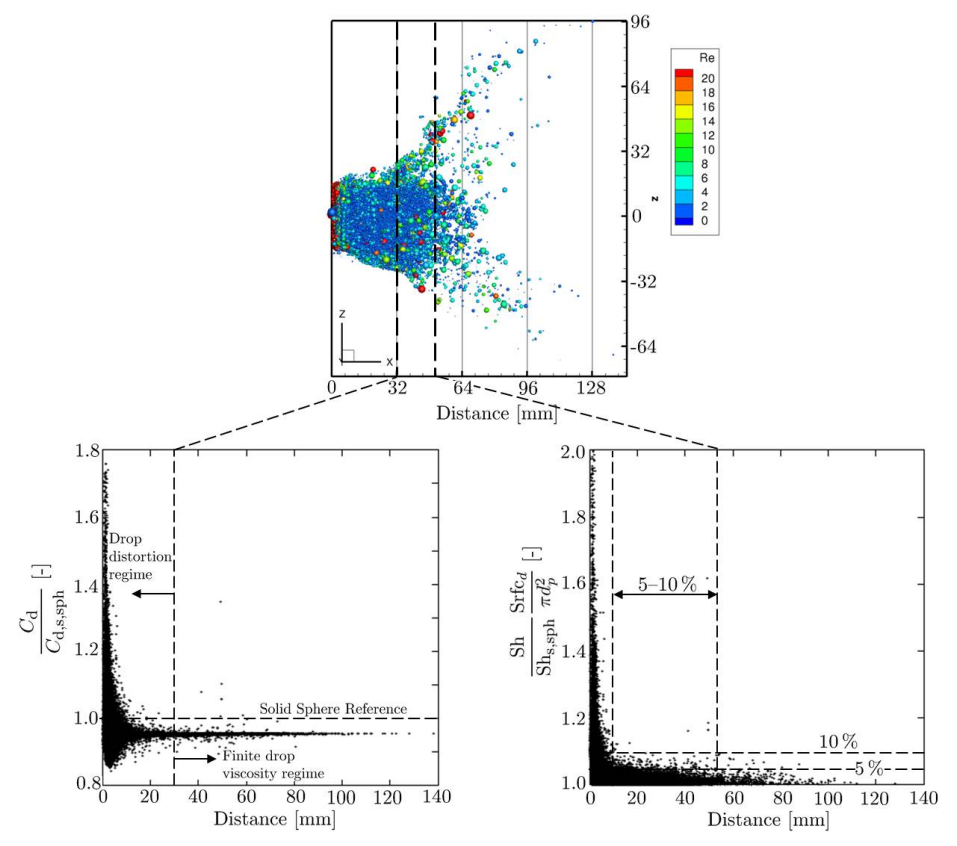

Figure 10: (Top) Visualization of the instantaneous spray distribution with drops color-coded by their drop Reynolds number. (Bottom, left) Effective drop drag coefficient correction calculated from Eq. (35). (Bottom, right) Drop evaporation rate calculated from Eqs (43).

lated from Eq. (35) (bottom, left); and the drop evaporation rate calculated from Eqs (43) (bottom, right). These results were obtained using a time step for Runge-Kutta integration selected based on the most restrictive characteristic time scale of the simulated physical processes (e.g., drop-flow momentum and drop oscillation). The liquid drops are color-coded by their local drop Reynolds number, established as the most meaningful flow field parameter. Figure 10 (bottom, left) shows the effective drop drag coefficient correction as a function of the spatial distance from the injector exit. This correction is using local drop conditions from the LES in combination with Eq. (35). The mapping process reveals that significant corrections due to drop deformations exist close to the injector exit $(x<10 \mathrm{~mm})$. This mapping also identifies the region of distinctive drop distortions $(\mathrm{x}<30 \mathrm{~mm})$, as highlighted in Fig. 6. At larger distances, the variation of drag coefficient corrections diminishes as a consequence of vanishing drop Reynolds numbers and Weber numbers. Figure 10 (bottom, right) shows the effective drop evaporation rates due to fluid-fluid interface dynamics, internal flow field patterns, shape distortion corrections to the Sherwood number, and surface area corrections in comparison to the solid sphere reference. The mapping process reveals that significant corrections $(5-10 \%)$ to the reference evaporation rate exist up to liquid penetration lengths of $\mathrm{x}<55 \mathrm{~mm}$. The analysis also shows that those corrections, in contrast to corresponding drag coefficient corrections, diminish in the limit 

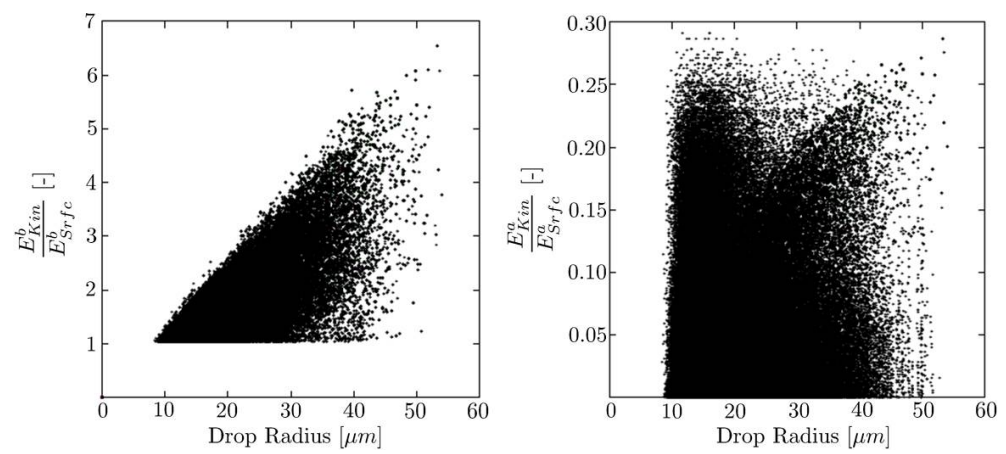

Figure 11: Ratio of kinetic energy to surface energy of drops before (left) and after (right) breakup calculated using Eqs. (69) and (72).

of vanishing drop Reynolds numbers and Weber numbers.

Having established predictions of drop and flow dynamics up to the point in time when breakup occurs, the following analysis focuses on the drop breakup event modeled by Eqs. (73), (77), (79), and (81). This set of equations provides the initial drop states after breakup while conserving mass, momentum, kinetic energy, and surface energy. The starting point focuses on the energy equation, which relates drop properties before breakup to the corresponding drop properties after breakup. The energy of the drops is computed from contributions of kinetic energy and surface energy, as defined in Eq. (69). Figure 11 (left) shows the ratio of energy contributions from drops before breakup as a function of the original drop radius conditioned on the time of critical deformation $(y=1)$. It is observed that, with increasing drop size, the contribution of the kinetic energy generally increases while the contribution of the surface energy diminishes. In contrast, Fig. 11 (right) shows the ratio of kinetic energy to surface energy of drops after breakup, as defined by Eq. (72). Compared to the state before breakup, the ratio of energy contributions has reversed. The breakup event has re-distributed the energy from a kinetic state to surface energy. Hence, the accurate calculation of surface energy is imperative for proper breakup modeling.

At first, the surface area of the deformed drop is calculated at the time of breakup from Eq. (61). This occurs when the drop poles meet at its center. Next, the surface tension force is computed from detailed mean-field molecular two-phase interface calculations and multiplied by the surface area to obtain the surface energy. Figure 12 (left) presents the ratio of Sauter mean radius predictions using Eq. (73) to predictions from the classic formulation proposed by O'Rourke and Amsden. ${ }^{75}$ Results from both models are calculated simultaneously during the simulation so that both models operate under the exact same conditions. The ratio of the two model predictions illustrates the significance 

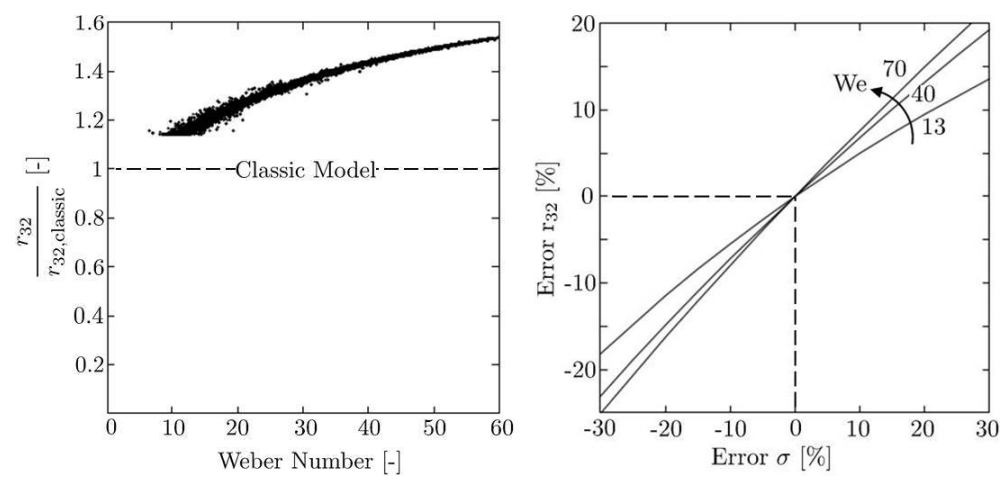

Figure 12: (Left) Ratio of Sauter mean radius obtained by the breakup model, defined in Eq. (73), to the original formulation proposed by O'Rourke and Amsden, ${ }^{75}$ conditioned on the Weber number at the time of breakup. (Right) Relation between errors in the computation of surface tension values and the resulting errors in the prediction of the Sauter mean radius with increasing Weber numbers.

of the new model refinements and the dependency on the Weber number. The new framework consistently predicts a larger Sauter mean radius of drops after breakup in comparison to the reference solution from O'Rourke and Amsden. It is noteworthy that many past studies have shown that the predicted drop sizes after breakup, obtained from the classic model, are often significantly underpredicted. ${ }^{48,55,104-106,109}$ The extent of the under-prediction reported in these studies is similar to the magnitude of the modifications which result from this work.

Figure 12 (right) quantifies resulting errors in predicted Sauter mean radius due to errors in the simulated value of surface tension forces. The analysis illustrates that a quasi-linear relationship exists between errors in surface tension forces and its corresponding impact on the predicted Sauter mean radius. The impact of errors in surface tension increases with the Weber number. It is noted that surface tension forces, even in high-fidelity simulations, are not necessarily calculated as rigorously as performed in this study. ${ }^{38,39,115}$ Hence, the error range assumed in Fig. 12 (right) appears conservative and highlights an opportunity for further improvements in future simulations.

The determination of the Sauter mean radius facilitates the calculation of the resulting drop size distribution based on the maximum entropy formalism (see Eq. (77)). After calculating the drop size distribution, the drop velocities after breakup are calculated. In addition to the parent drop velocity, each new drop is assigned a velocity in the normal direction as a function of the deformation rate of the parent drop. Figure 13 presents the normalized drop velocity and momentum distributions for illustration. Such distributions are shown in the $\eta-\zeta$ plane, which by definition is normal to the velocity vector of the original drop. The corresponding coordinate system is shown as an inset plot in Fig. 13 (a). The ratios of the circle radii represent the corresponding drop sizes exactly, as 

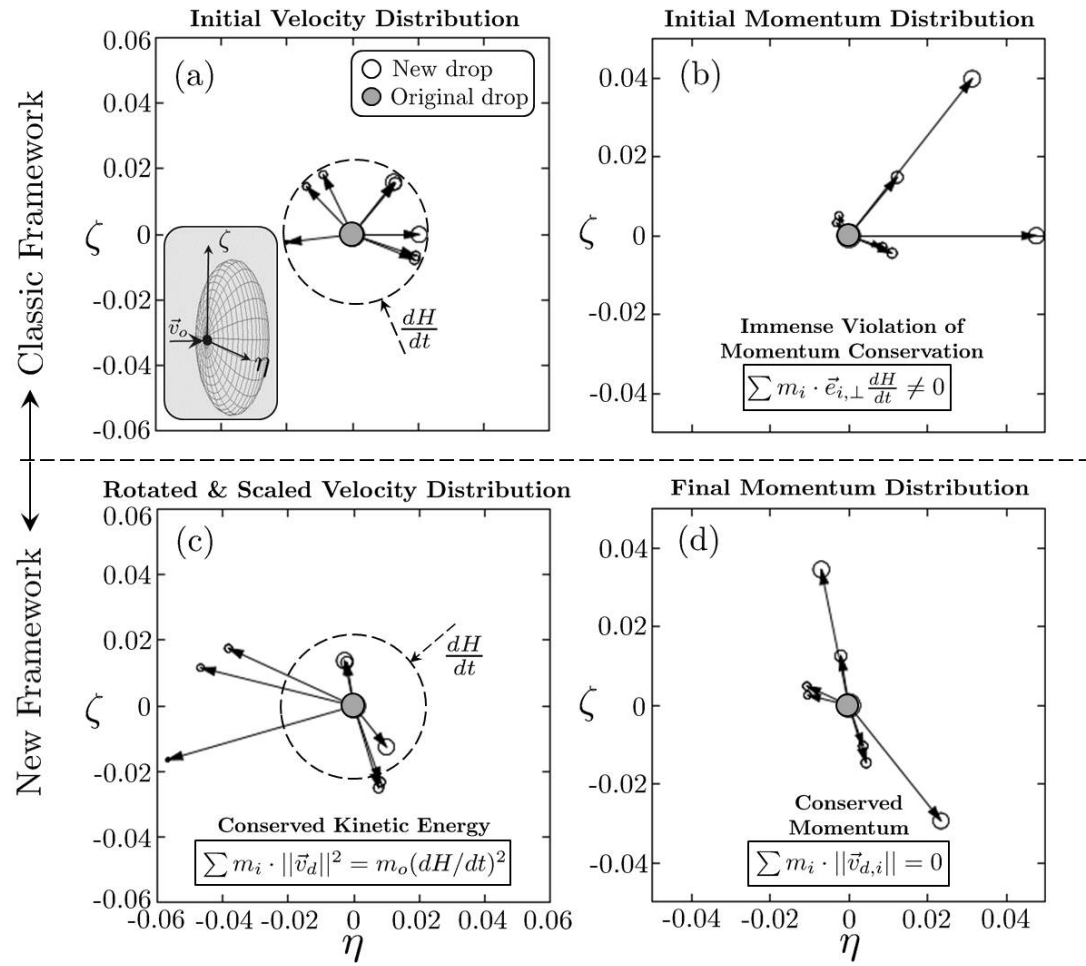

Figure 13: Normalized drop velocity and momentum distributions in the $\eta-\zeta$ plane normal to the velocity vector of the original drop (see inset plot). Symbols illustrate drop sizes of the original drop (grey circles) and the new drops (open circles). (a) Initial drop velocity distribution obtained using the random sampling method defined in Eq. (74). The velocity magnitude of each new drop is constant and equal to $\left\|\vec{v}_{d, \perp}\right\|=d H / d t$, as calculated from Eq. (67). (b) Corresponding momentum distribution after multiplication of the velocities in (a) with the respective drop masses, calculated from Eq. (77). Note that momentum contributions do not cancel and momentum conservation is dramatically violated as a consequence (see Eq. (76)). (c) Drop velocity distribution after rotation of the initial solution in (a) to enforce momentum conservation, as calculated from Eq. (80), and after subsequent scaling to maintain energy conservation, as defined by Eq. (81). (d) Conserved momentum distribution after the rotation and scaling operation. 
given by Eq. (77). The center (filled) circle represents the size of the parent drop before breakup for comparison. Figure 13 (a) shows the initial drop velocity distribution obtained using the random sampling method defined in Eq. (74). The velocity magnitude of each new drop is constant and equal to $\left\|\vec{v}_{d, \perp}\right\|=d H / d t$, as calculated from Eq. (67). This velocity distribution defines the corresponding drop momentum distribution, shown in Fig. 13 (b) when multiplied with the individual drop mass. Significant differences in drop mass lead to a heavily skewed momentum distribution. However, Eq. (76) requires that the momentum vectors must cancel out for momentum conservation. This is generally not done in current simulations. Figure 13 (b) explains why and illustrates a dramatic violation of momentum conservation. In contrast, the framework presented in this paper applies a unique rotation and scaling operation to enforce momentum and energy conservation during drop breakup, as defined in Eqs. (80) and (81). Figure 13 (c) and (d) show the corresponding drop velocity and momentum distributions after such corrections. The comparison to the initial solution illustrates the significance of the applied correction.

Given the observations above, the cumulative violation of momentum conservation is quantified for all breakup events that occur during the entire simulation. This is done by calculating the drop momentum for each breakup event, which is equal to the left-hand-side of Eq. (76). Recall that momentum conservation requires the left-hand-side to be zero. Figure 14 then shows the result of this calculation normalized by the reference momentum of the original parent drop; $p=m_{0} \cdot d H / d t$, as defined by Eqs. (75) and (67). Without explicit enforcement of momentum conservation in the calculation of the drop velocities, momentum conservation is consistently violated in a significant manner. The number of newly created drops, while depending on the computed ratio $r_{p} / r_{32}$, is generally insufficient to guarantee an approximate solution of Eq. (76) using statistical sampling. Furthermore, respective drop masses and drop velocities are chosen both randomly and independently of each other. As a consequence, a large number of drop samples would be required for a statistical solution of Eq. (76), which is not realizable. Consistent with this argument, Fig. 14 shows that the momentum conservation error decreases with an increase in Weber number due to the accompanying increase of generated drop numbers during breakup. The violation of momentum conservation never vanishes, which is unacceptable in a breakup model. In contrast, the new framework guarantees the exact conservation of momentum and energy for each breakup event. 


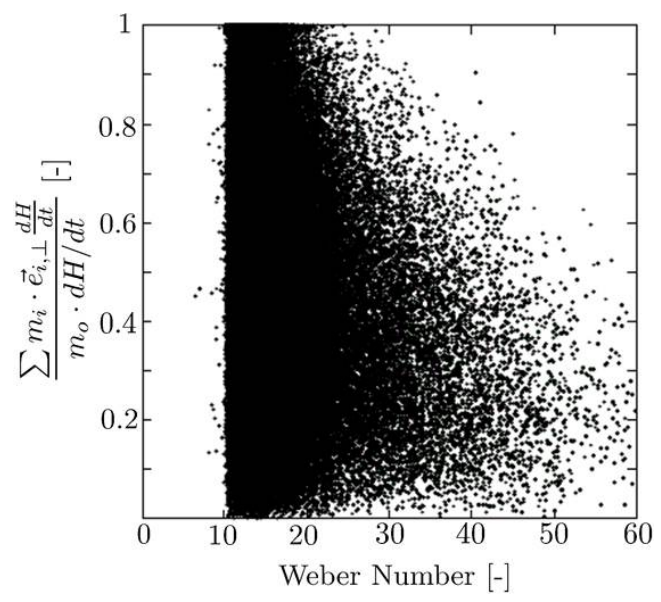

Figure 14: Error in momentum conservation at the time of breakup in the simulation as a function of the Weber number. The normal drop momentum is calculated from Eq. (76) and normalized by the drop reference momentum; $p=m_{0} \cdot d H / d t$, as defined in Eqs. (75) and (67). The calculations are performed using the classic method based on random sampling without explicit enforcement of momentum conservation.

\section{Summary and Conclusions}

Two classes of models have emerged over past decades for the simulation of atomization and breakup processes. The first is the classic WAVE model and the second is the Taylor Analogy Breakup (TAB) model. The TAB model is built on Taylor's analogy between drop dynamics and a forced springmass-damper system. It explicitly describes the deformation and oscillation of drop dynamics and assumes that drop breakup occurs as soon as oscillations of the drop poles meet at the drop center. The model is conceptually valid for a wide range of Ohnesorge and Weber numbers as long as surface stripping phenomena do not significantly contribute to the breakup process. Since its introduction, the original TAB model has motivated further improvements such as the Enhanced Taylor Analogy Breakup (ETAB) model. Despite these advancements, however, the atomization and breakup dynamics of liquid drops are not necessarily well described in current models.

In previously developed models for device-scale turbulent combustion simulations, the coupling of drop distortions, oscillations, and breakup as smaller-scale temporal fluctuations is largely neglected. The current work has focused on this potential deficiency by introducing a fundamentally-consistent framework suitable for Large Eddy Simulations to understand and quantify such potential errors on mass, momentum, and energy exchange functions. The framework utilizes real-fluid thermodynamics and transport along with Gradient Theory for accurate calculations of molecular two-phase interface exchange functions, surface tension forces, drop oscillations, and breakup processes. Aerodynamic forces 
were calculated using Large Eddy Simulations. A new set of equations were derived to improve the modeling of drop non-sphericity and drop breakup in sprays. The significance of these improvements to fuel injection processes was demonstrated in a model gas turbine combustor configuration.

The analysis presented demonstrates that negative drop distortions, which naturally occur during drop oscillations, significantly reduce drag forces. Furthermore, harmonic drag coefficient oscillations exhibit an exponential scaling with the drop distortion parameter. It was also established that the dynamic drag model, which assumes a sharp-edged disc as a reference shape at the critical deformation before breakup, generally significantly over-predicts the resulting drag forces as a consequence. Finally, effects from fluid-fluid interface dynamics and internal flow fields were shown to consistently reduce drag forces not accounted for in the dynamic drag model. Such effects were shown to persist over the full lifetime of the drop since they do not diminish as drops equilibrate with the flow.

The improved model framework quantifies the effects of transient deformation processes on evaporation and heating rates, which are largely neglected in modern simulations. It was demonstrated that the consideration of these coupling dynamics modify mass and energy transfer rates more significantly compared to the corresponding drag forces from momentum exchange. In contrast to drag forces, finite drop viscosity effects were demonstrated to generally amplify evaporation and heating transport phenomena. Such effects were shown to become diminished once the liquid drops equilibrate with the flow. An improved breakup model was derived from a refined energy balance equation that explicitly enforces drop momentum conservation during the breakup process. This in turn has shown that many previous breakup modeling frameworks inherently violate drop momentum conservation. The analysis established the significance of accurate predictions of surface tension forces in the breakup process, and a sensitivity analysis revealed a quasi-linear relationship between the fidelity of surface tension calculations and the corresponding accuracy of predicted Sauter mean diameter of drops after breakup. 


\section{Acknowledgement}

Support for this work was provided by Sandia National Laboratories under the Early Career LaboratoryDirected Research and Development (EC-LDRD) program. This research was also funded by the Division of Chemical Sciences, Geosciences and Biosciences, Office of Basic Energy Sciences, U.S. Department of Energy. Sandia National Laboratories is a multiprogram laboratory operated by Sandia Corporation, a Lockheed Martin Company, for the U.S. Department of Energy under contract DEAC04-AL85000. This research was performed at the Combustion Research Facility, Sandia National Laboratories, Livermore, California.

\section{References}

${ }^{1}$ Abramzon, B. and Sirignano, W. A. (1989). Droplet vaporization model for spray combustion calculations. Int. J. Heat Mass Transfer, 32:1605-1618.

${ }^{2}$ Amsden, A. A., O’Rourke, P. J., and Butler, T. D. (1989). Kiva ii: A computer program for chemically reactive flows with sprays. Technical Report LA-11560-MS, Los Alamos National Laboratory.

${ }^{3}$ Apte, S., Gorokhovski, M., and Moin, P. (2003). Les of atomizing spray with stochastic modeling of secondary breakup. Intl. J. Multiphase Flow, 20:1503-1522.

${ }^{4}$ Ashgriz, N. (2011). Handbook of Atomization and Sprays: Theory and Applications. Springer.

${ }^{5}$ Babinsky, E. and Sojka, P. E. (2002). Modeling drop size distributions. Progress Energy Combust. Sci., 28:303-329.

${ }^{6}$ Bhatt, Y., Arora, D., Shaw, R., and Golubev, V. (2011). Numerical simulations and performance comparison of air-blast and pressure jet atomizers. AIAA, 2011-277.

${ }^{7}$ Bird, R., Stewart, W., and Lightfoot, E. (1960). Transport Phenomena. John Wiley and Sons, Incorporated, New York.

${ }^{8}$ Brodkey, R. (1969). The Phenomena of Fluid Motions. Addison Wesley.

${ }^{9}$ Cahn, J. W. and Hilliard, J. E. (1958). Free energy of a nonuniform system. i. interfacial free energy. J. Chem. Phys., 28:258-267. 
${ }^{10}$ Chryssakis, C. and Assanis, D. N. (2008). A unified fuel spray breakup model for internal combustion engine applications. Atomization and Sprays, 18:1-52.

${ }^{11}$ Cousin, J., Yoon, S. J., and Dumouchel, C. (1996). Coupling of classical linear theory and maximum entropy formalism for prediction of drop size distribution in sprays: application to pressure-swirl atomizers. Atom. Sprays, 6:601-622.

12 Dahms, R. N. (2015). Gradient theory simulations of pure fluid interfaces using a generalized expression for influence parameters and a helmholtz energy equation of state for fundamentally consistent two-phase calculations. J. Colloid Interface Sci., 445:48-59.

${ }^{13}$ Dahms, R. N., Drake, M. C., Fansler, T. D., Grover, R. O., and Solomon, A. S. (2012). Detailed simulations of stratified ignition and combustion processes in a spray-guided gasoline engine using the SparkCIMM/G-equation modeling framework. SAE Int. J. Engines, 5:141-161.

${ }^{14}$ Dahms, R. N., Drake, M. C., Fansler, T. D., Kuo, T.-W., and Peters, N. (2011a). Understanding ignition processes in spray-guided gasoline engines using high-speed imaging and the extended spark-ignition model SparkCIMM. Part A: Spark channel processes and the turbulent flame front propagation. Combust. Flame, 158:2229-2244.

${ }^{15}$ Dahms, R. N., Drake, M. C., Fansler, T. D., Kuo, T.-W., and Peters, N. (2011b). Understanding ignition processes in spray-guided gasoline engines using high-speed imaging and the extended sparkignition model SparkCIMM. Part B: Importance of molecular fuel properties in early flame front propagation. Combust. Flame, 158:2245-2260.

${ }^{16}$ Dahms, R. N., Fansler, T. D., Drake, M. C., Kuo, T.-W., Lippert, A. M., and Peters, N. (2009). Modeling ignition phenomena in spray-guided spark-ignited engines. Proc. Combust. Inst., 32:27432750.

${ }^{17}$ Dahms, R. N., Felsch, C., Rohl, O., and Peters, N. (2011c). Detailed chemistry flamelet modeling of mixed-mode combustion in spark-assisted HCCI engines. Proc. Combust. Inst., 33:3023-3030.

${ }^{18}$ Drake, M. C., Fansler, T. D., and Lippert, A. M. (2005). Stratified-charge combustion: modeling and imaging of a spray-guided direct-injection spark-ignition engine. Proc. Combust. Inst., 30:2683-2691.

${ }^{19}$ Drake, M. C. and Haworth, D. C. (2007). Advanced gasoline engine development using optical diagnostics and numerical modeling. Proc. Combust. Inst., 31:99-124. 
${ }^{20}$ Eisenklam, P. and Arunachalam, S. (1966). The drag resistance of burning drops. Combust. Flame, 10.

${ }^{21}$ Eisenklam, P., Arunachalam, S., and Weston, J. (1967). Evaporation rates and drag resistance of burning drops. Eleventh Symposium (International) on Combustion, 11.

${ }^{22}$ Ely, J. and Hanley, H. (1981a). Prediction of transport properties. 1. viscosity of fluids and mixtures. Industrial and Engineering Chemistry Fundamentals, 20.

${ }^{23}$ Ely, J. and Hanley, H. (1981b). Prediction of transport properties. 2. thermal conductivity of pure fluids and mixtures. Industrial and Engineering Chemistry Fundamentals, 22.

${ }^{24}$ Erlebacher, G., Hussaini, M. Y., Speziale, C. G., and Zang, T. A. (1992). Toward the large eddy simulation of compressible turbulent flows. J. Fluid Mech., 238:155-185.

${ }^{25}$ Faeth, G. M. (1977). Current status of droplet and liquid combustion. Progress in Energy and Combustion Science, 3:191-224.

${ }^{26}$ Faeth, G. M. (1983). Evaporation and combustion in sprays. Progress in Energy and Combustion Science, 9:1-76.

${ }^{27}$ Faeth, G. M. (1987). Mixing, transport and combustion in sprays. Progress in Energy and Combustion Science, 13:293-345.

${ }^{28}$ Faeth, G. M. and Lazar, R. S. (1971). Fuel droplet burning rates in a combustion gas environment. AIAA Journal, 9:2165-2171.

${ }^{29}$ Fansler, T. D., Reuss, D. L., Sick, V., and Dahms, R. N. (2015). Combustion instability in sprayguided stratified-charge engines: A review. Int. J. Engine Res., 16:260-305.

${ }^{30}$ Feng, Z.-G. and Michaelides, E. (2001). Drag coefficents of viscous spheres at intermediate and high reynolds numbers. J. Fluids Engineering, 123:841-849.

${ }^{31}$ Germano, M., Piomelli, U., Moin, P., and Cabot, W. H. (1991). A dynamic subgrid-scale eddy viscosity model. Phys. Fluids, 3:1760-1765.

${ }^{32}$ Gordon, S. and McBride, B. (1971). Computer Program for Calculation of Complex Chemical Equilibrium Compositions, Rocket Performance, Incident and Reflected Shocks and Chapman-Jouguet Detonations. NASA SF-273. 
${ }^{33}$ Gorokhovski, M. and Herrmann, M. (2008). Modeling primary atomization. Annu. Rev. Fluid Mech., 40:343-366.

${ }^{34}$ Gorokhovski, M. and Saveliev, V. (2003). Analyses of kolmogorov's model of breakup and its application into lagrangian computation of liquid sprays under air-blast atomization. Phys. Fluids, $15: 184-192$.

${ }^{35}$ Gorokhovski, M. and Saveliev, V. (2008). Statistical universalities in fragmentation under scaling symmetry with a constant frequency of fragmentation. J. Physics D: Applied Physics, 41:085405.

${ }^{36}$ Haider, A. and Levenspiel, O. (1989). Drag coefficient and terminal velocity spherical and nonspherical particles. Powder Technol., 58:63-70.

${ }^{37}$ Helenbrook, B. and Edwards, C. (2002). Quasi-steady deformation and drag of uncontaminated liquid drops. Int. J. Multiphase Flow, 28:1631-1657.

${ }^{38}$ Herrmann, M. (2010). Detailed numerical simulations of the primary atomization of a turbulent liquid jet in crossflow. J. Engineering Gas Turbines Power, 132:061506.

${ }^{39}$ Herrmann, M. (2011). The influence of density ratio on the primary atomization of a turbulent liquid jet in crossflow. Proc. Combust. Inst., 33:2079-2088.

${ }^{40}$ Hirschfelder, J., Curtiss, C. F., and Bird, R. B. (1964). Molecular Theory of Gases and Liquids. John Wiley and Sons, Incorporated, New York.

${ }^{41}$ Hölzer, A. and Sommerfeld, M. (2008). New simple correlation formula for the drag coefficient of non-spherical particles. Powder Technol., 184:361-365.

${ }^{42}$ Hsiang, L.-P. and Faeth, G. (1995). Drop deformation and breakup due to shock wave and steady disturbances. Int. J. Multiphase Fow, 21:545-560.

${ }^{43} \mathrm{Hu}$, B., Musculus, M. P. B., and Oefelein, J. C. (2012). The influence of large-scale structures on entrainment in a decelerating transient turbulent jet revealed by large eddy simulation. Phys. Fluids, 24:045106-1-17.

${ }^{44}$ Huang, C. and Lipatnikov, A. (2011). Modelling of gasoline and ethanol hollow-cone sprays using openfoam. SAE Technical Paper, 2011-01-1896. 
${ }^{45}$ Hubbard, G., Denny, V., and Mills, A. (1975). Droplet evaporation: Effects of transients and variable properties. Int. J. Heat Mass Transfer, 18:1003-.

${ }^{46}$ Joseph, D. D., Belanger, J., and Beavers, G. S. (1999). Breakup of a liquid drop suddenly exposed to a high-speed airstream. Int. J. Multiphase Flow, 25:1263-1303.

${ }^{47}$ Kee, R., Rupley, F., and Miller, J. (1990). Chemkin Thermodynamic Data Base. SAND87-8215B.

${ }^{48}$ Kumzerova, E. and Esch, T. (2008). Extension and validation of the cab droplet breakup model to a wide weber number range. Institute for Liquid Atomization and Spray Systems, 2008-4-5.

${ }^{49}$ Lamb, H. (1932). Hydrodynamics. Cambridge University Press.

${ }^{50}$ Lasheras, J. C. and Hopfinger, E. J. (2000). Liquid jet instability and atomization in a coaxial gas stream. Annu. Rev. Fluid Mech., 32:275-308.

${ }^{51}$ Law, C. K. (1982). Recent advances in droplet vaporization and combustion. Progress in Energy and Combustion Science, 8:171-201.

${ }^{52}$ Leland, T. W. and Chappelear, P. S. (1968). The corresponding states principle. a review of current theory and practice. Industrial and Engineering Chemistry Fundamentals, 60:15-43.

${ }^{53}$ Lilly, D. K. (1992). A proposed modification of the germano subgrid-scale closure method. Phys. Fluids, 3:633-635.

${ }^{54}$ Lin, H., Duan, Y.-Y., and Min, Q. (2007). Gradient Theory modeling of surface tension for pure fluids and binary mixtures. Fluid Phase Equil., 254:75-90.

${ }^{55}$ Liu, A., Mather, D., and Reitz, R. (1993a). Modeling the effects of drop drag and breakup on fuel sprays. SAE Technical Paper, 930072.

${ }^{56}$ Liu, A. B., Mather, D., and Reitz, R. D. (1993b). Modeling the effects of drop drag and breakup on fuel sprays. SAE Technical Paper, 930072.

${ }^{57}$ Manimaran, R. and Raj, R. T. K. (2014). Computational studies of swirl ratio and injection timing on atomization in a direct injection diesel engine. Frontiers in Heat and Mass Transfer, 5:1-9.

${ }^{58}$ Marmottant, P. and Villermaux, E. (2004). On spray formation. J. Fluid Mech., 498:73-111. 
${ }^{59}$ McCoy, B. F. and Davis, H. T. (1979). On the free energy theory of inhomogeneous fluids. Phys. Rev. A, 20:1201-1207.

${ }^{60}$ Mejia, A., Pamies, J. C., Duque, D., Seguara, H., and Vega, L. F. (2005). Phase and interface behaviors and type-i and type-v lennard-jones mixtures: Theory and simulations. J. Chem. Phys., $123: 1-10$.

${ }^{61}$ Miller, R., Harstad, K., and Bellan, J. (1998). Evaluation of equilibrium and non-equilibrium evaporation models for many-droplet gas-liquid flow simulations. Int. J. Multiphase Flow, 24:10251055.

${ }^{62}$ Miqueu, C., Mendiboure, B., and Lachaise, J. (2005). Modeling of the surface tension of multicomponent mixtures with the gradient theory of fluid interfaces. Ind. Eng. Chem. Res., 44:3321-3329.

${ }^{63}$ Miqueu, C., Miguez, J. M., Pineiro, M. M., Lafitte, T., and Mendiboure, B. (2011). Simultaneous application of the gradient theory and monte carlo molecular simulation for the investigation of methane/water interfacial properties. J. Phys. Chem. B, 115:9618-9625.

${ }^{64}$ Moin, P., Squires, K., Cabot, W., and Lee, S. (1991). A dynamic subgrid-scale model for compressible turbulence and scalar transport. Phys. Fluids, 3:2746-2757.

${ }^{65}$ Moiz, A. A., Som, S., Bravo, L., and Lee, S.-Y. (2015). Experimental and numerical studies on combustion model selection for split injection spray combustion. SAE Technical Paper, 2015-01-0374.

${ }^{66}$ Muller, E. A. and Mejia, A. (2009). Interfacial properties of selected binary mixtures containing n-alkanes. Fluid Phase Equilibria, 282:68-91.

${ }^{67}$ Oefelein, J. (2006a). Large eddy simulation of turbulent combustion processes in propulsion and power systems. Progress in Aerospace Sciences, 42:2-37.

${ }^{68}$ Oefelein, J. C. (2005). Thermophysical characteristics of lox-h2 flames at supercritical pressure. Proc. Combust. Inst., 30:2929-2937.

${ }^{69}$ Oefelein, J. C. (2006b). Mixing and combustion of cryogenic oxygen-hydrogen shear-coaxial jet flames at supercritical pressure. Combust. Sci. Tech., 178:229-252.

${ }^{70}$ Oefelein, J. C., Dahms, R. N., and Lacaze, G. (2012). Detailed modeling and simulation of highpressure fuel injection processes in diesel engines. SAE Intl. J. Engines, 5:1410-1419. 
${ }^{71}$ Oefelein, J. C., Lacaze, G., Dahms, R. N., Ruiz, A., and Misdariis, A. (2014). Effects of real fluid thermodynamics in high-pressure fuel injection processes. SAE Intl. J. Engines, 7:1125-1136.

${ }^{72}$ Oefelein, J. C., Sankaran, V., and Drozda, T. G. (2007). Large eddy simulation of swirling particleladen flow in a model axisymmetric combustor. Proc. Combust. Inst., 31:2291-2299.

${ }^{73}$ Oefelein, J. C., Schefer, R. W., and Barlow, R. W. (2006). Toward validation of les for turbulent combustion. AIAA Journal, 44:418-433.

${ }^{74}$ Okong'o, N. and Bellan, J. (2004). Consistent large-eddy simulation of a temporal mixing layer laden with evaporating drops. part 1. direct numerical simulation, formulation and a priori analysis. J. Fluid Mech., 499:1-47.

${ }^{75}$ O'Rourke, P. and Amsden, A. (1987). The tab method for numerical calculation of spray droplet breakup. SAE Technical Paper, 872089.

${ }^{76}$ Putnam, A. (1961). Integratable form of droplet drag coefficient. ARS Journal, 31:1467-1468.

${ }^{77}$ Ramanujan, S. (1914). Modular equations and approximations to pi. Quart. J. Pure App. Math, $45: 350-372$.

${ }^{78}$ Ranz, W. E. and Marshall, W. R. (1952). Evaporation from drops. Chemical Engineering Progress, 48:141-173.

${ }^{79}$ Reid, R. C., Prausnitz, J. M., and Polling, B. E. (1987). The Properties of Liquids and Gases. McGraw-Hill, New York.

${ }^{80}$ Reitz, R. D. (1987). Modelling atomization processes in high-pressure vaporizing sprays. Atomization and Spray Technology, 3:309-337.

${ }^{81}$ Reitz, R. D. and Bracco, F. V. (1982). Mechanism of atomization of a liquid jet. Phys. Fluids, 25:1730-1742.

${ }^{82}$ Reitz, R. D. and Diwakar, R. (1987). Structure of high-pressure fuel sprays. SAE Paper, 870598.

${ }^{83}$ Ricart, L. M., Xin, J., Bower, G. R., and Reitz, R. D. (1997). In-cylinder measurement and modeling of liquid fuel spray penetration in a heavy-duty diesel engine. SAE Technical Paper, 971591. 
${ }^{84}$ Richter, A. and Nikrityuk, P. (2012). Drag forces and heat transfer coefficients for spherical, cuboidal and ellipsoidal particles in cross flow at sub-critical reynolds numbers. Int. J. Heat Mass Transfer, $55: 1343-1354$.

${ }^{85}$ Rowlinson, J. S. (1979). Translation of j. d. van der waals' "the thermodynamic theory of capillarity under the hypothesis of a continuous variation of density. J. Stat. Phys, 20:200-244.

${ }^{86}$ Rowlinson, J. S. and Watson, I. D. (1969). The prediction of the thermodynamic properties of fluids and fluid mixtures-i. the principle of corresponding states and its extensions. Chem. Eng. Sci., $24: 1565-1574$.

${ }^{87}$ Ruiz, A. M., Lacaze, G., and Oefelein, J. C. (2015). Flow topologies and turbulence scales in a jet-in-cross-flow. Phys. Fluids, 27:045101.

88 Sazhin, S. S. (2006). Advanced models of fuel droplet heating and evaporation. Prog. Energy Combust. Sci., 32:162-214.

${ }^{89}$ Schmidt, K. A. G., Folas, G. K., and Kvamme, B. (2007). Calculation of the interfacial tension of methane-water system with the linear gradient theory. Fluid Phase Equil., 261:230-237.

90 Senecal, P., Pomraning, E., Richards, K., and Som, S. (2013). An investigation of grid convergence for spray simulations using an les turbulence model. SAE Technical Paper, 2013-01-1083.

${ }^{91}$ Senecal, P. K., Richards, K. J., Pomraning, E., Yang, T., Dai, M. Z., McDavid, R. M., Patterson, M. A., Hou, S., and Shethaji, T. (2007). A new parallel cut-cell cartesian cfd code for rapid grid generation applied to in-cylinger diesel engine simulations. SAE Technical Paper, 2007-01-0159.

92 Sirignano, W. (1999). Fluid Dynamics and Transport of Droplets and Sprays. Cambridge Uni. Press.

93 Sirignano, W. A. (1983a). Fuel droplet vaporization and spray combustion theory. Prog. Energy Combust. Sci., 9:291-322.

${ }^{94}$ Sirignano, W. A. (1983b). Fuel droplet vaporization and spray combustion theory. Progress in Energy and Combustion Science, 9:291-322.

${ }^{95}$ Smagorinsky, J. (1963). General circulation experiments with the primitive equations. I. The basic experiment. Monthly Weather Review, 91:99-164. 
96 Som, S. and Aggarwal, S. K. (2009). Assessment of atomization models for diesel engine simulations. Atom. Sprays, 19:885-903.

${ }^{97}$ Sommerfeld, M., Ando, A., and Wennerberg, D. (1992). Swirling, particle-laden flows through a pipe expansion. Journal of Fluids Engineering, 114:648-656.

98 Sommerfeld, M. and Qiu, H.-H. (1991). Detailed measurements in a swirling particulate two-phase flow by a phase-doppler anemometer. International Journal of Heat and Fluid Flow, 12(1):20-28.

99 Sommerfeld, M. and Qiu, H.-H. (1993). Characterization of particle-laden, confined swirling flows by phase-doppler anemometry and numerical calculation. International Journal of Multiphase Flow, 19(6):1093-1127.

100 Spalding, D. B. (1955). Some Fundamentals of Combustion. Butterworths, London.

101 Spalding, D. B. (1959). Theory of particle combustion at high pressure. ARS Journal, 29:828-835.

102 Speziale, C. G. (1985). Galilean invariance of subgrid-scale stress models in the large eddy simulation of turbulence. J. Fluid Mech., 156:55-62.

103 Takahashi, S. (1974). Preparation of a generalized chart for the diffusion coefficients of gases at high pressures. Journal of Chemical Engineering of Japan, 7:417-420.

104 Tanner, F. (1997). Liquid jet atomization and droplet breakup modeling of non-evaporating diesel fuel sprays. SAE Technical Paper, 970050.

105 Tanner, F. (2003). A cascade atomization and drop breakup model for the simulation of highpressure liquid jets. SAE Technical Paper, 2003-01-1044.

106 Tanner, F. and Weisser, G. (1998). Simulation of liquid jet atomization for fuel sprays by means of a cascade drop breakup model. SAE Technical Paper, 980808.

107 Taylor, G. (1963). The shape and acceleration of a drop in a high speed air stream, volume 3 . In G.K. Batchelor, ed., The scientific papers of Sir Geoffrey Ingram Taylor, Cambridge University Press. 108 Trinh, H. and Chen, C. (2006). Modeling of turbulence effects on liquid jet atomization and breakup. Atomization Spray, 16:097-932. 
109 Trinh, H., Chen, C., and Balasubramanyam, M. (2005). Numerical simulation of liquid jet atomization including turbulence effects. 41st AIAA/ASME/SAE/ASEE Joint Propulsion Conference, 2005-3973.

110 Turner, M. R., Sazhin, S. S., Healey, J. J., Crua, C., and Martynov, S. B. (2012). A breakup model for transient diesel fuel sprays. Fuel, 97:288-305.

${ }^{111}$ van der Waals, J. D. (1893). Square gradient model. Verhandel Konik Akad Weten Amsterdam, $1: 8-15$.

${ }^{112}$ VanWylen, G. J. and Sonntag, R. E. (1986). Fundamentals of Classical Thermodynamics. John Wiley and Sons, Incorporated, New York.

${ }^{113}$ Vreman, B., Geurts, B., and Kuerten, H. (1994). On the formulation of the dynamic mixed subgridscale model. Phys. Fluids, 6:4057-4059.

${ }^{114}$ Wang, Z., Swantek, A., Scarcelli, R., Duke, D., Kastengren, A., Powell, C. F., Som, S., Reese, R., Freeman, K., and Zhu, Y. (2015). Les of diesel and gasoline sprays with validation against x-ray radiography data. SAE Int. J. Fuels Lubr., 8.

${ }^{115}$ Xiao, F., Dianat, M., and McGuirk, J. J. (2014). Les of turbulent liquid jet primary breakup in turbulent coaxial flow. Int. J. Multiphase Flow, 60:103-118.

${ }^{116}$ Yang, X., Solomon, A., and Kuo, T.-W. (2012). Ignition and combustion simulations of sprayguided sidi engine using arrhenius combustion with spark-energy deposition model. SAE Technical Paper, 2012-01-0147.

${ }^{117}$ Yuen, M. and Chen, L. (1976). On drag of evaporating liquid droplets. Combust. Sci. Techn., 14:147-154.

${ }^{118}$ Zang, Y., Street, R. L., and Koseff, J. R. (1993). A dynamic mixed subgrid-scale model and its application to turbulent recirculating flows. Phys. Fluids, 5:3186-3195.

${ }^{119}$ Zuo, Y.-X. and Stenby, E. H. (1996). A linear gradient theory model for calculating interfacial tensions of mixtures. J. Colloid Interface Sci., 182:126-132. 